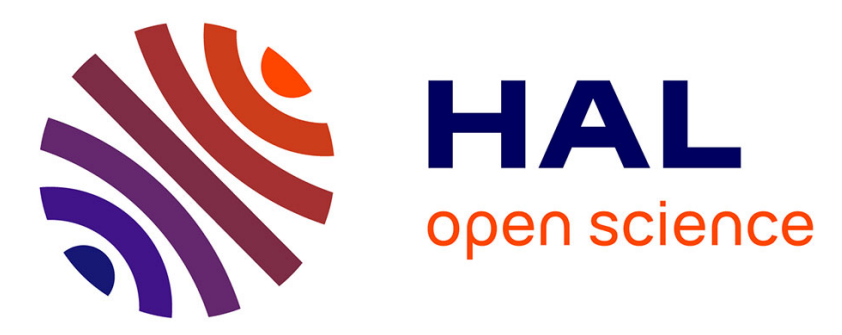

\title{
Numerical simulations and modeling of the effective plastic flow surface of a biporous material with pressurized intergranular voids
}

Guylaine Boittin, Pierre-Guy Vincent, Hervé Moulinec, Mihail Garajeu

\section{- To cite this version:}

Guylaine Boittin, Pierre-Guy Vincent, Hervé Moulinec, Mihail Garajeu. Numerical simulations and modeling of the effective plastic flow surface of a biporous material with pressurized intergranular voids. Computer Methods in Applied Mechanics and Engineering, 2017, 323, pp.174 - 201. 10.1016/j.cma.2017.05.004 . hal-01653321

\section{HAL Id: hal-01653321 \\ https://hal.science/hal-01653321}

Submitted on 4 Dec 2017

HAL is a multi-disciplinary open access archive for the deposit and dissemination of scientific research documents, whether they are published or not. The documents may come from teaching and research institutions in France or abroad, or from public or private research centers.
L'archive ouverte pluridisciplinaire HAL, est destinée au dépôt et à la diffusion de documents scientifiques de niveau recherche, publiés ou non, émanant des établissements d'enseignement et de recherche français ou étrangers, des laboratoires publics ou privés. 


\title{
Numerical simulations and modeling of the effective plastic flow surface of a biporous material with pressurized intergranular voids
}

\author{
Guylaine Boittin $^{\mathrm{a}, \mathrm{b}}$, Pierre-Guy Vincent ${ }^{\mathrm{a}, \mathrm{c}, *}$, Hervé Moulinec ${ }^{\mathrm{b}}$, Mihai Garajeu ${ }^{\mathrm{b}}$ \\ ${ }^{a}$ Institut de Radioprotection et de Sûreté Nucléaire, B.P. 3, 13115 Saint-Paul-lez-Durance Cedex, France \\ ${ }^{b}$ Aix Marseille Univ, CNRS, Centrale Marseille, LMA, Marseille, France, UPR 7051, F-13402 Marseille \\ Cedex 20, France \\ ${ }^{c}$ Laboratoire de Micromécanique et Intégrité des Structures, IRSN-CNRS-UM, B.P. 3, 13115 \\ Saint-Paul-lez-Durance Cedex, France
}

\begin{abstract}
This study is devoted to the effective plastic flow surface of a biporous polycrystalline material, with an intragranular porosity consisting of spherical voids, and an intergranular porosity consisting of larger elongated voids along the grain boundaries. These two populations of voids (or bubbles) with well separated scales and shapes are saturated by a fluid and therefore are subjected to internal pressures. The effect of the intragranular voids is modeled through a GTN (Gurson-Tvergaard-Needleman) criterion in the matrix. Numerical simulations are performed with a FFT-based (Fast Fourier Transforms) method. A particular attention is paid to the effect of the distribution of the intergranular bubbles on the effective plastic flow surface. Different microstructures with different volume fractions and sizes for the intergranular bubbles are tested under three loading conditions (the mean size of the grains being fixed). Two main results are exhibited. First, it is shown that the effect of the relative size of the intergranular bubbles on the effective plastic flow surface depends on the loading direction. Secondly, a comparison is made with the analytical model of (Vincent et al., 2014) and a correction of the porosity relative to the intergranular bubbles is introduced in this analytical model in order to take into account the specific distribution of the intergranular bubbles along the grain boundaries. This correction is expressed as a sum of two power law functions, each of them being significant either for low or for large values of the porosity of intergranular bubbles.
\end{abstract}

Keywords: Porous materials, Effective plastic flow surface, Micromechanical models, Intergranular cavities, Microstructures, Numerical algorithms

${ }^{*}$ Corresponding author. Tel: +334421997 61; fax: +33442199166

Email addresses: guylaine.boittin@irsn.fr (Guylaine Boittin), pierre-guy.vincent@irsn.fr (Pierre-Guy Vincent), moulinec@lma.cnrs-mrs.fr (Hervé Moulinec), garajeu@lma.cnrs-mrs.fr (Mihai Garajeu) 


\section{Introduction}

This study is devoted to the effective plastic flow surface of a polycrystalline material exhibiting two populations of voids: spherical voids which are located inside the grains, and larger elongated voids along the grain boundaries. The intragranular voids are randomly distributed inside the grains and are much smaller than the intergranular voids. Moreover, these two populations of voids (or bubbles) are saturated by a fluid and therefore are subjected to internal pressures. These bubbles may have a strong influence on the mechanical behavior. The aim of this work is to improve an existing analytical model for the effective plastic flow surface of such a biporous saturated material Vincent et al. (2014a,b). Such a model is useful in specific engineering problems where the plasticity must take into account the dilatational effects due to the presence of bubbles.

As an example of a real material, we can cite the irradiated uranium dioxide $\left(\mathrm{UO}_{2}\right)$, which exhibits, as a first approximation, such a specific microstructure. $\mathrm{UO}_{2}$ is commonly used as fuel in nuclear reactors and its mechanical behavior is a topic of continuous research in order to estimate the safety of nuclear reactors. The effects of irradiation on the uranium dioxide fuel are complex. The irradiation leads to the creation of defects (vacancies, interstitials), and gazeous fission products (such as xenon and krypton). The interaction of vacancies and fission gas atoms leads to the formation of two populations of bubbles: micrometric intergranular bubbles with a lenticular form, and smaller, almost spherical, intragranular bubbles. The reader is referred to (Kashibe and Une, 1991; Kashibe et al., 1993; Lösönen, 2000) in order to get more information about the formation and the size of these voids. As they retain gases, the bubbles are pressurized. Their volume, shape and pressure evolve with temperature and irradiation. Several studies in micromechanics have been devoted to the influence of bubbles on $\mathrm{UO}_{2}$ mechanical behavior (Vincent et al., 2008, 2009a,b; Julien et al., 2011; Vincent et al., 2014a,b).

Vincent et al. (2009a,b, 2014a,b) have investigated the effective plastic flow surface of a material with a bimodal population of pressurized bubbles. In these studies, the intragranular behavior is considered as already pre-homogenized: the effect of pressurized intragranular bubbles is described through a Gurson-Tvergaard-Needleman (GTN) plasticity criterion (Gurson, 1977; Koplik and Needlemann, 1988; Tvergaard, 1990). Vincent et al. (2014a,b) have derived a simple analytical expression for the effective plastic flow surface and its accuracy has been assessed by comparison with full-field numerical simulations. In order to improve the accuracy of this model, the present study investigates the effect on the effective plastic flow surface of a distribution of lenticular bubbles on and along the grain boundaries in a Gurson-like material. The main difference with the previous study, is not the shape of the bubbles, ellipsoidal versus lenticular, but the more realistic distribution of the bubbles along the grain boundaries, instead of randomly distributed and oriented bubbles. In both cases, the microstructures are macroscopically isotropic: randomly oriented and distributed ellipsoidal bubbles in an isotropic matrix in (Vincent et al., 2014a,b), lenticular bubbles on and along randomly oriented fictitious grain boundaries 
in an isotropic matrix for the present study. The key point is that, here, the lengths of the lenticular bubbles are smaller than the lengths of the fictitious grains, so that several bubbles can be located along the same grain boundary. It leads to specific microstructures with strings of bubbles along the grains boundaries (as typically observed in irradiated $\mathrm{UO}_{2}$ ). During a loading, the local fields such as the plastic strain rate, can be strongly influenced by the distribution of the voids inside the matrix. The objective is then to catch the effect of such a specific distribution of bubbles on the effective (or macroscopic) plastic flow surface.

The present paper is organized as follows. The second section discuses the pertinent microstructural parameters with a particular attention paid to those concerning the shape and the size of the voids. The third section details the modeling of the microstructure. The fourth section exhibits the numerical tools to simulate the mechanical behavior of periodic cells with a Fast Fourier Transforms method (Michel et al., 2001; Moulinec and Silva, 2014). Section 5 shows the test matrix (different sets of parameters describing the microstructure and the loadings), the results of the calculations and their analysis. Then, in section 6 , the analytical model of the previous study of Vincent et al. (2014a,b) is recalled, from which a corrected porosity is designed to take into account the specific distribution of the intergranular bubbles.

\section{Microstructural data}

\subsection{General microstructure}

The materials addressed in this study can be considered as bi-porous materials characterized by the presence of two populations of voids with well separated sizes: (1) spherical intragranular bubbles at a very small scale, and (2) elongated (along grain boundaries) intergranular bubbles at a larger scale (Figure $1 \mathrm{~b}$ )). As the scales of the two populations are well separated, the effective plastic flow surface of such a material can be obtained by two successive homogenization steps as in (Vincent et al., 2014b):

- the first step, corresponding to the scale of the intragranular bubbles, by the way of a Gurson-like plasticity criterion,

- the second step, corresponding to the scale of the intergranular bubbles, via full-field numerical simulations based on a FFT (Fast Fourier Transforms) method.

The main originality of the study is the distribution of voids along the grain boundaries. The effect of this distribution is of interest in several situations. Two main examples can be cited : materials exhibiting intergranular ductile fracture may present such intergranular porosities and irradiated materials.

Nuclear fuel pellet of $\mathrm{UO}_{2}$ was taken as an example to determine microstructural parameters close a real case : 
$\mathrm{UO}_{2}$ is, at the microscopical level, a polycrystal with a typical grain size of about 10 $\mu \mathrm{m}$, (Killeen, 1980; Kashibe and Une, 1991; Kashibe et al., 1993; Dherbey et al., 2002). The nuclear reaction creates rare gases such as xenon and krypton, which are trapped in the material and can form pressurized bubbles inside the grains or at the grain boundaries. Intragranular bubbles are typically considered as almost spherical in shape and their radii can be of the order of nanometer in some observations by transmission electron microscopy (TEM) and could reach a few hundred of nanometers in a fuel submitted to a strong irradiation test (power ramp) (Kashibe et al., 1993; Lösönen, 2000). Intergranular bubbles have a lenticular form, elongated along the grain boundaries, due to enhanced diffusion of vacancies and gases atoms through the grain boundaries.

Figure 1 a) shows a microstructure obtained by Scanning Electron Microscopy (SEM) of an irradiated $\mathrm{UO}_{2}$ (Dubourg et al., 2005) illustrating bubbles sizes, morphologies and localization.

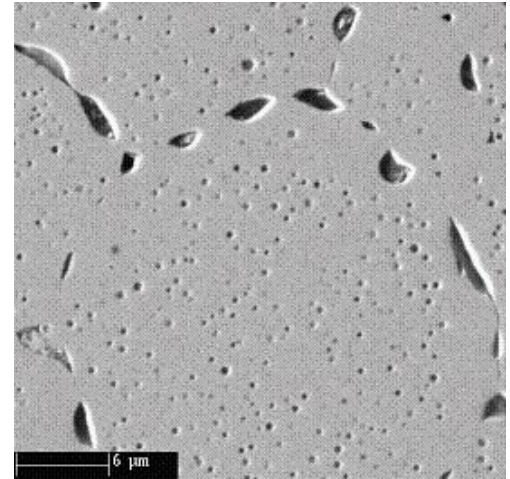

a)

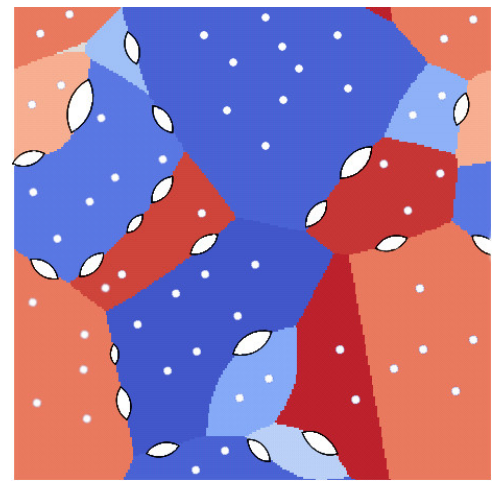

b)

Figure 1: (a) Microstructure of a $23 \mathrm{GWd} / \mathrm{t}$ irradiated $\mathrm{UO}_{2}$ after a heat treatment (temperature holding 3 hours at $2000 \mathrm{~K}$ ) (Dubourg et al., 2005). (b) Idealized microstructure (for FFT simulations).

\subsection{Intergranular bubbles}

Several microstructural parameters are necessary in order to describe accurately the intergranular cavities. Like in many studies, the intergranular bubbles are characterized by the porosity $f_{e}$ (volume fraction of the intergranular voids) and by their shape, size and distribution.

As mentioned, the intergranular cavities are elongated along grain boundaries, but their distribution is not described by a scalar or a tensorial parameter. It derives directly from the position of the grains boundaries.

In the present study, a broad range of porosity $(0.1$ to $6 \%)$ is investigated in order to determine the effects of the volume fraction. At high volume fractions, bubbles may 
form an interconnected network. According to White (2004), in $\mathrm{UO}_{2}$ fuel intergranular bubbles interlinkage begins to occur when the fractional coverage of the grain boundaries exceeds $17-18 \%$ in agreement with statistical models of coalescence on plane surfaces. In the present study, it is chosen to limit the analysis to the non-interconnected bubbles, even for high fractional coverages. For the sake of simplicity, the bubbles located at triple point (at the intersection of three or more grains) were also neglected.

For a more realistic representation, it is supposed that the intergranular cavities have a lenticular shape (see Figure 2 and Appendix A). This is in line with the observations of Reynolds et al. (1971), Hodkin (1980), Une (1988) and Zacharie et al. (1998) realized by TEM and/or SEM on a $\mathrm{UO}_{2}$ fuel. The lenticular shape is characterized by the contact angle, $\theta$, between the grain boundary plan and the surface of the bubble. Physically, the contact angle depends on the surface energy of the bubble and on the grain boundary energy (Reynolds et al., 1971). These energies themselves depend on misorientation between the grains. Therefore, the contact angle is not the same from one grain boundary to another. For example, Reynolds et al. (1971) found values between $34^{\circ}$ and $52^{\circ}$ in the same sample. A fixed value of the contact angle is classically chosen to simulate the bubbles, and they are considered to have all the same shape. In this study a fixed value of $45^{\circ}$ for the contact angle is set in the following.

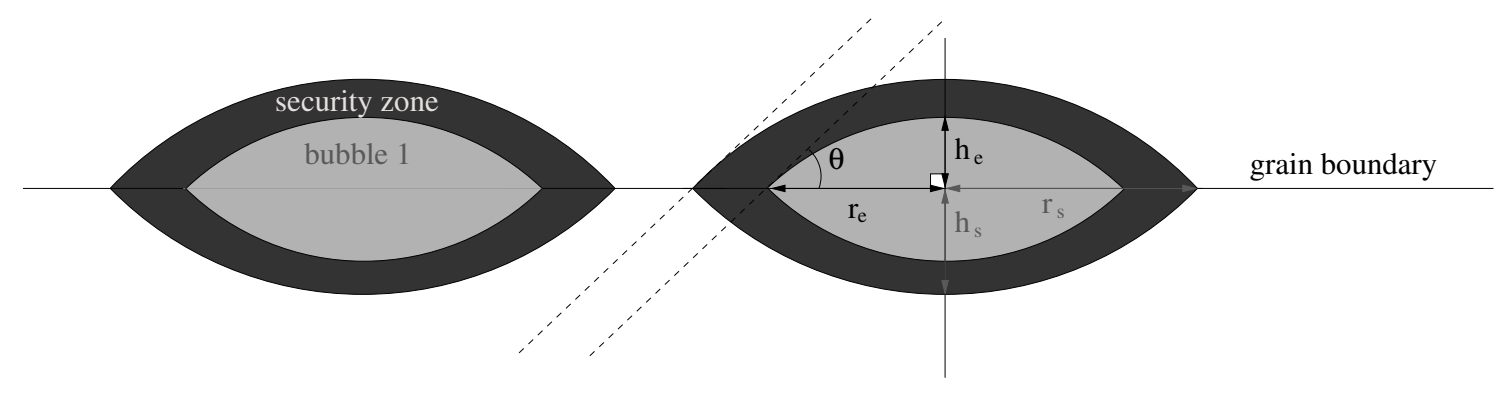

Figure 2: Notations for the intergranular bubbles. Definition of the security zones around the intergranular bubbles.

The size of intergranular cavities can vary a lot from one cavity to another. For lenticular shapes it is characterized by the radius $r_{e}$ (Figure 2 and Appendix A) and by the height $h_{e}=r_{e} \tan \frac{\theta}{2}$ of the lenticular zone. Few bibliographic results give information on the populations of cavities. But, Noirot et al. (2004) give very precise results for a $\mathrm{UO}_{2}$ LWR fuel irradiated at $61 \mathrm{GWd} / \mathrm{t}$. The measured intragranular and intergranular porosities are around $1 \%$, the diameter of the intergranular bubbles is comprised between 100 $\mathrm{nm}$ and $1 \mu \mathrm{m}$ with a mean value around $700 \mathrm{~nm}$ and the diameter of intragranular bubbles is around $300 \mathrm{~nm}$. In the present study, the relative sizes of the bubbles are chosen to avoid spatial resolution problems and therefore correspond only to the biggest observed bubbles.

The microstructural parameters used for our numerical study are summarized in Table 
1.

\begin{tabular}{ccccc} 
Bubble & Parameter & Notation & Min & Max \\
\hline Intragranular & Local porosity inside the grains & $f_{b}$ & $0.7 \%$ & $6 \%$ \\
\hline Intergranular & Porosity & $f_{e}$ & $0.1 \%$ & $6 \%$ \\
& Relative radius (on the grain boundary) & $r_{e} / r_{g}$ & 0.1 & 0.32 \\
& Contact angle & $\theta$ & $45^{\circ}$ & $45^{\circ}$ \\
& Normalized minimal distance & $r_{s} / r_{e}$ & 1.25 & 1.75
\end{tabular}

Table 1: Microstructural parameters for the simulations. The mean radius of the grains is denoted by $r_{g}$.

\section{Microstructure modeling}

The porosity due to the intragranular bubbles is taken into account through a GTN model (Gurson, 1977; Koplik and Needlemann, 1988; Tvergaard, 1990). Only the intergranular bubbles are geometrically discretized in $V$, the representative volume element (RVE). In the simulations, this volume is considered as a cube with a length $L$ and the mean radius of the grains $r_{g}$ is defined as:

$$
\frac{r_{g}}{L}=\left(\frac{3}{4 \pi N}\right)^{1 / 3},
$$

where $N$ denotes the number of grains in $V$.

As specified in section 2.2, the intergranular bubbles are elongated along the grain boundaries and have a lenticular form. Let us define a simple grain boundary as the boundary between exactly two grains, and the triple boundary as the boundary between three or more grains. Here, the intergranular bubbles have the following characteristics:

- located only on simple grain boundaries,

- lenticular with a long axis along the grain boundary,

- with identical size $\left(r_{e}\right)$ and shape (same contact angle, $\theta$ ).

An algorithm has been developed for this specific microstructure. Its main concept is to mimic the growth of the bubbles along the grain boundaries. The microstructure is generated in four successive steps:

$i$ generate a polycrystal with a Voronoi tesselation,

ii identify the grain boundaries, 
iii generate random seeds for the bubbles on the grain boundaries,

$i v$ let the bubbles grow until they reach their final size.

In particular, the fourth step consists in making easier the growth of the bubbles along the grain boundaries rather than inside the grains. For each bubble, the growth is stopped when the desired radius $r_{e}$ is reached. This process tends to create bubbles with the desired final radius along the grain boundary $\left(r_{e}\right)$ and the desired contact angle $(\theta)$. The general process is illustrated in Figure 3.

Moreover, to avoid overlapping between the bubbles, a security radius $\left(r_{s}\right)$ has been introduced. The growth of the security zones follows the same algorithm as the one for the bubbles growth with the same seeds, the same shape (same aspect ratio $w_{l}=h_{s} / r_{s}=h_{e} / r_{e}$, see Figure 2), but with a size of $r_{s}$ instead of $r_{e}$. Two security zones of two distinct bubbles can not overlap. So this process not only avoids overlapping between the bubbles but also ensures a minimal distance between two bubbles centers along a grain boundary of $2 r_{s}$ $\left(r_{s} \geq r_{e}\right)$ in the microstructure, as illustrated in Figure 2 .

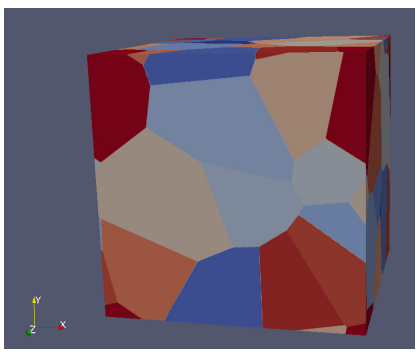

Polycristal Voronoi tesselation

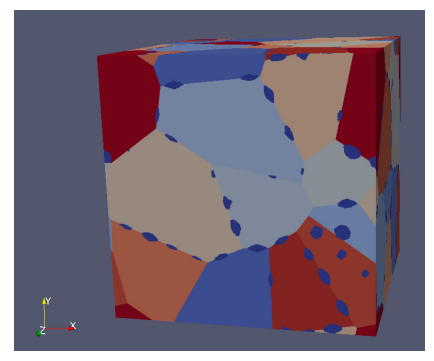

Elongated voids along the grain boundaries

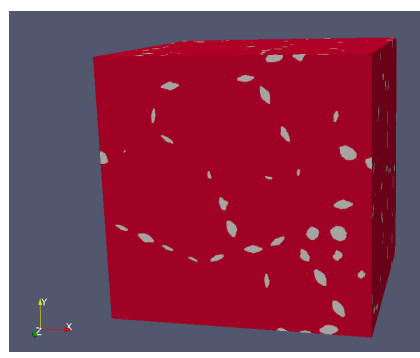

Final two-phases microstructure

Figure 3: Procedure for the generation of the microstructures.

\section{Numerical determination of the effective flow surface}

\subsection{FFT method}

Full-field numerical simulations are performed using the CraFT software as presented in Suquet et al. (2012). The software is based on the FFT method initially proposed in Moulinec and Suquet $(1994,1998)$ to investigate the effective properties of periodic composites. The first algorithm developed on that basis, called in this article "basic algorithm", was described in Moulinec and Suquet (1998) and Michel et al. (2001). This iterative method is based on the exact expression of the Green function for a linear elastic, homogeneous reference material. To improve the convergence of this algorithm on composites with high contrasts, some "accelerated schemes" were derived from the basic one, for example 
the augmented Lagrangian (Michel et al., 2000), the Eyre-Milton algorithm (Eyre and Milton, 1999), or the Monchiet-Bonnet algorithm (Monchiet and Bonnet, 2012). Moulinec and Silva (2014) have proven that the augmented Lagrangian and the Eyre-Milton algorithm are particular cases of the Monchiet-Bonnet algorithm (the values of the scalar parameters $\alpha$ and $\beta$ in equation (3) are equal to 1 for augmented Lagrangian and to 2 for the Eyre-Milton algorithm). Moreover, the FFT method was extended to elasto-viscoplastic composites using a step-by-step time integration (Idiart et al., 2006). This method can be readily used in accelerated schemes.

The method works as follows: at each time step, a fixed point algorithm is used to determine the local strain and stress fields (respectively denoted by $\varepsilon$ and $\boldsymbol{\sigma}$ ). The fixed point algorithm for the basic and accelerated schemes can be written by the following set of equations (Monchiet and Bonnet, 2012; Idiart et al., 2006). At each iteration $i, \boldsymbol{\sigma}^{i}, \boldsymbol{\varepsilon}^{i}$ are known from the last iteration and $\boldsymbol{\sigma}^{i+1}, \boldsymbol{\varepsilon}^{i+1}$ are determined as follows:

Basic scheme:

$$
\left\{\begin{array}{l}
\varepsilon^{i+1}=\varepsilon^{\mathrm{i}}-\boldsymbol{\Gamma}^{0} * \boldsymbol{\sigma}^{i} \\
\boldsymbol{\sigma}^{i+1}=f\left(\varepsilon^{i+1}, v_{\text {int }}\right)
\end{array}\right.
$$

Accelerated scheme:

$$
\left\{\begin{array}{l}
\boldsymbol{\tau}^{\mathrm{i}+1}=\boldsymbol{\tau}^{\mathrm{i}}-\alpha \boldsymbol{C}_{0}: \boldsymbol{\Gamma}^{0} * \boldsymbol{\sigma}^{i}-\beta \boldsymbol{\Delta}^{0} * \boldsymbol{\varepsilon}^{i}-\beta \boldsymbol{C}_{0}:\left(<\boldsymbol{\varepsilon}^{\mathrm{i}}>-\boldsymbol{E}\right) \\
\boldsymbol{\sigma}^{i+1}=f\left(\varepsilon^{i+1}, v_{i n t}\right) \\
\boldsymbol{\tau}^{i+1}=\boldsymbol{\sigma}^{i+1}+\boldsymbol{C}_{0}: \boldsymbol{\varepsilon}^{i+1}
\end{array}\right.
$$

where $\langle$.$\rangle is the spatial average, \boldsymbol{E}$ is the macroscopic deformation, $\boldsymbol{C}_{0}$ is the stiffness tensor of the reference material, $\Gamma^{0}$, the Green operator associated to $\boldsymbol{C}_{0}, \boldsymbol{\Delta}^{0}$, the so-called "stress Green's tensor" (Kröner, 1972; Bhattacharya and Suquet, 2005), $v_{\text {int }}$ is a vector of internal variables, and $f$ is a general function relative to the constitutive relations.

For both schemes, the first equation (in (2) and (3)) ensures the global equilibrium of the system, and the second equation (in (2) and (3)) ensures the respect of the constitutive relations at each point. It is worth noting that the accelerated scheme does not require to solve $\boldsymbol{\sigma}=f\left(\varepsilon, v_{\text {int }}\right.$ ) anymore (where the known tensor is the local strain $\varepsilon$ and the unknown is $\boldsymbol{\sigma})$, but the set $\left(\boldsymbol{\tau}=\boldsymbol{\sigma}+\boldsymbol{C}_{0}: \boldsymbol{\varepsilon}, \boldsymbol{\sigma}=f\left(\varepsilon, v_{\text {int }}\right)\right.$ ) (where the known tensor is the polarization $\boldsymbol{\tau}$ and the unknowns are $\boldsymbol{\sigma}$ and $\boldsymbol{\varepsilon}$ ).

\subsection{Time-integration of the constitutive relations}

The matrix (the domain surrounding the intergranular bubbles) is an isotropic elastic perfectly plastic material obeying a GTN criterion (Gurson, 1977; Koplik and Needlemann, 1988; Tvergaard, 1990). As an uniform internal pressure $P_{b}$ is prescribed in the bubbles, the general form of the Gurson surface is preserved, with a shift of $P_{b}$ along the hydrostatic axis (Vincent et al., 2009a):

$$
F(\boldsymbol{\sigma})=q_{3}\left(\frac{\sigma_{e q}}{\sigma_{0}}\right)^{2}+2 q_{1} f_{b} \cosh \left(\frac{3\left(\sigma_{m}+P_{b}\right)}{2 \sigma_{0}}\right)-1-\left(q_{1} f_{b}\right)^{2},
$$


where $f_{b}$ is the local intragranular porosity; $\sigma_{0}$ is the yield stress of the unvoided material; $\sigma_{e q}$ is the Von Mises stress; $\sigma_{m}$ the hydrostatic stress; $\sigma_{m}=\frac{1}{3} \operatorname{Tr}(\boldsymbol{\sigma}) ; \sigma_{e q}=\sqrt{\frac{3}{2} \boldsymbol{\sigma}_{D}: \boldsymbol{\sigma}_{D}}$; $\boldsymbol{\sigma}_{D}=\boldsymbol{\sigma}-\sigma_{m} \boldsymbol{i} ; \boldsymbol{i}$ is the second order identity tensor and $q_{1}, q_{3}$ are two scalars introduced by Koplik and Needlemann (1988) and Tvergaard (1990) for a better match to numerical results.

For the purpose of this study, the values of $q_{1}$ and $q_{3}$ have been fixed to 1 (as in the original Gurson criterion). This choice is motivated by the following considerations. First, one should remark that the effect of $q_{1}$ is to enhance the effect of local porosity $f_{b}$ in the criterion. As the effect of $f_{b}$ on the final result of the present analysis is especially studied in section 6.3.1, the parameter $q_{1}$ is here fixed to 1 without loss of generality. Secondly, an expression for $q_{3}$ has been proposed by Leblond et al. (1994) in order to satisfy the upper bound of Ponte Castañeda (1991) for a purely deviatoric loading: $q_{3}=1+\frac{2}{3} f_{b}$. As the calculations of the present analysis are performed with moderate values for $f_{b}$ (less than 6 $\%$ ), this expression for $q_{3}$ remains close to 1 . For the sake of simplicity, $q_{3}$ has been fixed to 1 in the present analysis.

It is worth noting that our main objective is to derive the instantaneous effective plastic flow surface in the sense of Ponte Castañeda and Zaidman (1996). Therefore, the microstructure is supposed to be fixed: there is neither evolution of the porosities nor of the geometry of the microstructure. As a result, using the small strain hypothesis, the constitutive law for the matrix reads as:

$$
\left\{\begin{array}{l}
\dot{\boldsymbol{\sigma}}=\boldsymbol{C}:\left(\dot{\boldsymbol{\varepsilon}}-\dot{\boldsymbol{\varepsilon}}_{p}\right) \\
\dot{\boldsymbol{\varepsilon}}_{p}=\dot{\lambda} \frac{\partial F}{\partial \boldsymbol{\sigma}} \\
\dot{\lambda} \geq 0, F(\boldsymbol{\sigma}) \leq 0, \quad \dot{\lambda} F(\boldsymbol{\sigma})=0
\end{array}\right.
$$

where $\boldsymbol{C}$ is the elastic tensor of the porous matrix, $\varepsilon_{p}$ is the plastic part of the strain, and $\lambda$ is the plastic multiplier. For the time integration of the constitutive equations, the strain increment $(\dot{\varepsilon} \delta t)$ is supposed to be known. To use a radial return method (Wilkins, 1964), let us define a trial stress, $\boldsymbol{\sigma}^{T}$, as $\boldsymbol{\sigma}^{T}=\boldsymbol{\sigma}^{t_{0}}+\boldsymbol{C}: \dot{\boldsymbol{\varepsilon}} \delta t$, where $\boldsymbol{\sigma}^{t_{0}}=\boldsymbol{\sigma}(t-\delta t)$ is the known value of the stress at the previous time step. The algorithm for solving the set of equations (5) can be written as:

- compute the trial stress,

- if $F\left(\boldsymbol{\sigma}^{T}\right)<0$, then $\boldsymbol{\sigma}=\boldsymbol{\sigma}^{T}$,

- otherwise, $\boldsymbol{\sigma}$ is determined by solving the set of equations (5), where the last line is replaced by $F(\boldsymbol{\sigma})=0$. It leads to the following set of equations: 


$$
\left\{\begin{array}{l}
\sigma_{m}=\sigma_{m}^{T}-9 k f_{b} \frac{\lambda}{\sigma_{0}} \sinh \left(\frac{3\left(\sigma_{m}+P_{b}\right)}{2 \sigma_{0}}\right) \\
\boldsymbol{\sigma}_{D}=\boldsymbol{\sigma}_{D}^{T}-6 \frac{\mu \lambda}{\sigma_{0}^{2}} \boldsymbol{\sigma}_{D} \\
\left(\frac{\sigma_{e q}}{\sigma_{0}}\right)^{2}+2 f_{b} \cosh \left(\frac{3\left(\sigma_{m}+P_{b}\right)}{2 \sigma_{0}}\right)-1-f_{b}^{2}=0 .
\end{array}\right.
$$

The bulk and shear moduli for the elastic part are denoted by $k$ and $\mu$. The plastic multiplier, $\lambda$, can be expressed as a function of the hydrostatic stress from the first equation of $(6)$ :

$$
\lambda=\left(\frac{\sigma_{m}^{T}-\sigma_{m}}{9 k f_{b} \sinh \left(\frac{3\left(\sigma_{m}+P_{b}\right)}{2 \sigma_{0}}\right)}\right) \sigma_{0} .
$$

The second line of the set (6) shows that the deviatoric part of the trial stress and the deviatoric part of the stress are collinear. Therefore this equation reduces to a scalar equation for the equivalent stress:

$$
\sigma_{e q}=\left(\frac{\sigma_{0}^{2}}{\sigma_{0}^{2}+6 \mu \lambda}\right) \sigma_{e q}^{T} .
$$

Replacing the expression of $\lambda$ and $\sigma_{e q}$ in the last line of (6) shows that the set of equations (6) is then reduced to one scalar equation with one scalar unknown, $\sigma_{m}$ :

$$
\left(3 k \sigma_{e q}^{T} G\left(\sigma_{m}\right)\right)^{2}+\left(3 k \sigma_{0} G\left(\sigma_{m}\right)+2 \mu\left(\sigma_{m}^{T}-\sigma_{m}\right)\right)^{2} H\left(\sigma_{m}\right)=0
$$

with:

$$
\begin{gathered}
G\left(\sigma_{m}\right)=f_{b} \sinh \left(\frac{3\left(\sigma_{m}+P_{b}\right)}{2 \sigma_{0}}\right), \\
H\left(\sigma_{m}\right)=2 f_{b} \cosh \left(\frac{3\left(\sigma_{m}+P_{b}\right)}{2 \sigma_{0}}\right)-1-f_{b}^{2} .
\end{gathered}
$$

Once $\sigma_{m}$ has been determined, $\lambda$ is computed from equation (7) and $\boldsymbol{\sigma}_{D}$ is computed from the second line of (6). The stress $\boldsymbol{\sigma}$ is then fully determined.

Equation (9) is numerically solved. It has been observed that the function to nullify is close to a parabola in the vicinity of the solution. Therefore, the Müller method (Müller, 1956), based on parabola roots search seems well adapted to solve this equation. Note that the condition $(\lambda>0)$ is equivalent to the condition $\left(\sigma_{m}\right.$ comprised between $-P_{b}$ and $\left.\sigma_{m}^{T}\right)$. This bounding condition is used to initialize the Müller method.

The integration in the FFT scheme is straightforward concerning the basic scheme, by setting $\dot{\varepsilon} \delta t=\varepsilon^{i+1}-\varepsilon^{t_{0}}$ and $\boldsymbol{\sigma}=\boldsymbol{\sigma}^{i+1}$ (where $\varepsilon^{t_{0}}$ is the known value of the strain at the previous time step). For the accelerated scheme, the integration of the constitutive 
relations has to be rewritten following the same approach and introducing the increment of polarization $\dot{\boldsymbol{\tau}} \delta t=\boldsymbol{\tau}^{i+1}-\boldsymbol{\tau}^{t_{0}}$ (where $\boldsymbol{\tau}^{t_{0}}$ is the known value of the polarization at the previous time step). The direction of $\boldsymbol{\sigma}_{D}$ is obtained from the equation:

$$
\boldsymbol{\sigma}_{D}^{i+1}\left(1+\frac{\mu}{\mu_{0}}+\frac{6 \mu \lambda}{\sigma_{0}^{2}}\right)=\frac{\mu}{\mu_{0}}\left(\tau_{D}^{i+1}-\tau_{D}^{t_{0}}\right)+\left(1+\frac{\mu}{\mu_{0}}\right) \boldsymbol{\sigma}_{D}^{t_{0}}
$$

and the non-linear equation to solve (as a function of $\sigma_{m}^{i+1}$ ) reads as:

$$
\frac{3}{2}\left(\frac{3 k G\left(\sigma_{m}^{i+1}\right)}{3 k \sigma_{0}\left(1+\frac{\mu}{\mu_{0}}\right) G\left(\sigma_{m}^{i+1}\right)+2 \mu L\left(\sigma_{m}^{i+1}\right)}\right)^{2} M+H\left(\sigma_{m}^{i+1}\right)=0,
$$

with:

$$
\begin{gathered}
L\left(\sigma_{m}^{i+1}\right)=\frac{k}{k_{0}}\left(\tau_{m}^{i+1}-\tau_{m}^{t_{0}}\right)+\left(1+\frac{k}{k_{0}}\right)\left(\sigma_{m}^{t_{0}}-\sigma_{m}^{i+1}\right) \\
M=\left(\frac{\mu}{\mu_{0}}\left(\boldsymbol{\tau}_{D}^{i+1}-\boldsymbol{\tau}_{D}^{t_{0}}\right)+\left(1+\frac{\mu}{\mu_{0}}\right) \boldsymbol{\sigma}_{D}^{t_{0}}\right):\left(\frac{\mu}{\mu_{0}}\left(\boldsymbol{\tau}_{D}^{i+1}-\boldsymbol{\tau}_{D}^{t_{0}}\right)+\left(1+\frac{\mu}{\mu_{0}}\right) \boldsymbol{\sigma}_{D}^{t_{0}}\right)
\end{gathered}
$$

where $\left(k_{0}, \mu_{0}\right)$ are the bulk and shear moduli of the reference material. This equation is solved as for the basic scheme equation, by the mean of the Müller method. Finally, the equations (7) and (12) lead to the stress $\boldsymbol{\sigma}^{i+1}$. The strain $\varepsilon^{i+1}$ is then obtained through $\varepsilon^{i+1}=\boldsymbol{C}_{0}^{-1}:\left(\boldsymbol{\tau}^{i+1}-\boldsymbol{\sigma}^{i+1}\right)$. In the next section, numerical simulations are performed by means of the augmented Lagrangian accelerated scheme, in order to solve the elasto-plastic problem of a porous phase in a matrix governed by a GTN criterion (without evolution of the porosities nor of the geometry of the microstructure).

Following Vincent et al. (2014b), the effective flow surface can be characterized, in the present context, as the overall stress states obtained as asymptotic limits of the average stress along specific loading paths. Moreover, the effective extremal surface does not depend here on the type of plasticity theory, incremental or deformation theory, used for the individual constituents. In Vincent et al. (2014b), a deformation theory has been used, whereas, in the present article, an incremental theory has been adopted. But here, in the present context, both models will lead asymptoticaly to the same effective flow surface.

\section{Numerical full-field simulations}

\subsection{Spatial resolution}

Five unit cells are considered to calibrate the spatial resolution of the numerical simulations. Each cell is a cubic structure containing a lenticular void at its center. They are designed such that the cells have the same porosity $(2 \%)$ and the same shape parameter $\left(w_{l}=0.5\right.$, see Appendix A). The matrix is an elastic perfectly plastic material with a Gurson criterion such as described in section 4.2 , with $k=28 \mathrm{GPa}, \mu=21 \mathrm{GPa}, \sigma_{0}=1 \mathrm{GPa}, f_{b}=$ 
$2 \%$ and $P_{b}=0$. The cubic cells corresponds to distinct discretizations into $46^{3}, 64^{3}, 128^{3}$, $256^{3}$, and $512^{3}$ voxels. Each cell is tested under two loading conditions: a simple traction test condition along the direction of the revolution axis of the void $\left(\Sigma_{11} \neq 0, \Sigma_{22}=0\right.$, $\left.\Sigma_{33}=0, \Sigma_{i j, i \neq j}=0\right)$ and a purely hydrostatic test $\left(\Sigma_{11}=\Sigma_{22}=\Sigma_{33}, \Sigma_{i j, i \neq j}=0\right)$. Note that, in this work, only the direction of the overall stress (or macroscopic stress $\boldsymbol{\Sigma}$ ) is prescribed following a procedure described in (Michel et al., 1999) and (Michel et al., 2000).

The relative error (with respect to the highest resolution) on the macroscopic stress $\Sigma_{11}$ are plotted in Figure 4. The error is lower than $2.2 \%$ for the lowest discretization. In the following work, a minimal value of 2000 voxels for the discretization of one bubble is adopted, leading to a good compromise between size and accuracy (it would correspond here to a cell with $10^{5}$ voxels).

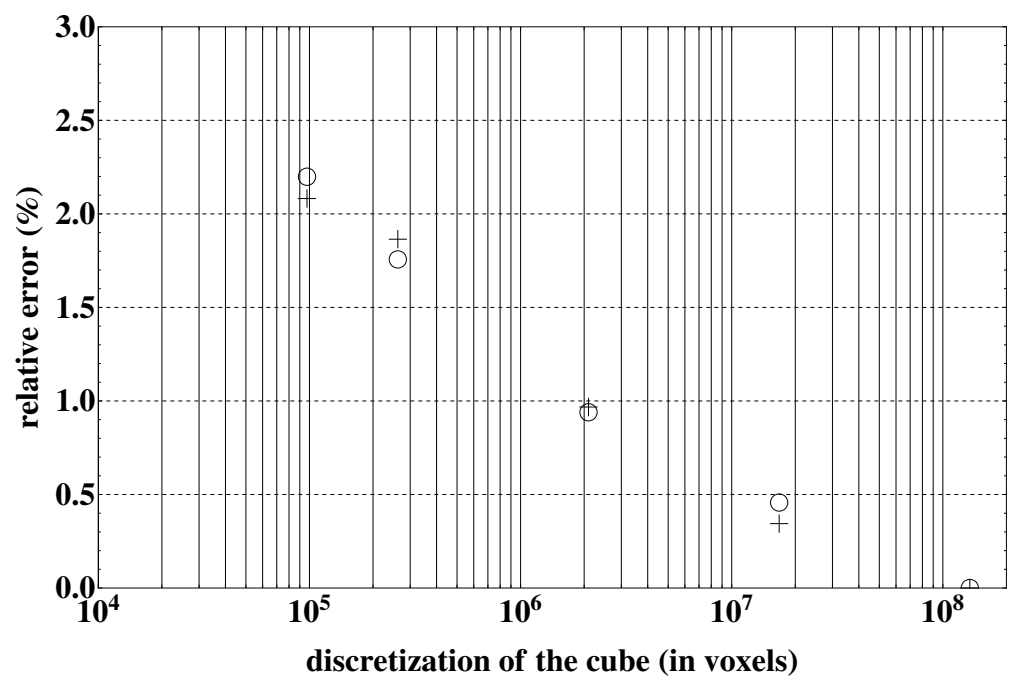

Figure 4: Single lenticular bubble void in a cubic cell. Influence of the spatial resolution. Relative error (with respect to highest resolution) on the macroscopic stress $\Sigma_{11}$ for two loading conditions: simple traction (circles) and purely hydrostatic traction (crosses) and five discretizations of the cell.

\subsection{Volume elements and loadings}

\subsubsection{Microstructural parameters}

In order to limit the size of the calculations, the discretization of the periodic cells is limited to $512^{3}$ voxels. For a cell with 80 grains, 2000 voxels per bubble means that the minimal relative size for the bubbles is around 0.16 (relative with respect to the mean grain size). To investigate smaller bubbles, a second cell is generated with 21 grains. In this second cell, the minimal relative size for the bubbles is 0.1 . Several values for the porosity 
of the intergranular bubbles, $f_{e}$, and for the radius of the bubble projected on the grain boundaries, $r_{e}$, and for the security radius, $r_{s}$, are tested. The test matrix is summarized in tables 2 and 3. Saturated grain boundaries means that the number of the bubbles is not prescribed, but that the bubbles are randomly drawn one by one until no more bubbles can be added (with respect to the security zones, as described in section 3). In this process, the bubbles are not moved or redrawn in order to increase their number. The test matrix leads to a total amount of 25 sets of parameters for the microstructures. Two examples of microstructures are shown on Figure 5.

\begin{tabular}{|c|c|c|c|c|}
\hline grains & \multicolumn{3}{|c|}{$r_{e} / r_{g}$} & $f_{e}(\%)$ \\
\hline \multirow{2}{*}{21} & 0.1 & 0.16 & 0.2 & 0.1 \\
\cline { 2 - 5 } & 0.1 & 0.16 & 0.2 & 1 \\
\hline \multirow{2}{*}{80} & 0.16 & \multicolumn{2}{|c|}{0.32} & 0.1 \\
\cline { 2 - 5 } & 0.16 & 0.32 & 1 \\
\hline
\end{tabular}

Table 2: Test matrix for the microstructures with $r_{s} / r_{e}=1.75$ and a controlled volume fractions $f_{e}$.

\begin{tabular}{|c|c|c|c|c|c|c|c|c|c|c|}
\hline grains & \multicolumn{10}{|c|}{$r_{e} / r_{g}$} \\
\hline \multirow{3}{*}{21} & & \multicolumn{3}{|c|}{0.1} & \multicolumn{3}{|c|}{0.16} & \multicolumn{3}{|c|}{0.2} \\
\hline & $r_{s} / r_{e}$ & 1.25 & 1.5 & 1.75 & 1.25 & 1.5 & 1.75 & 1.25 & 1.5 & 1.75 \\
\hline & $f_{e}(\%)$ & 3.42 & 2.38 & 1.76 & 4.18 & 2.87 & 2.11 & 4.40 & 3.13 & 2.24 \\
\hline \multirow{3}{*}{80} & & \multicolumn{5}{|c|}{0.16} & \multicolumn{4}{|c|}{0.32} \\
\hline & $r_{s} / r_{e}$ & \multicolumn{2}{|c|}{1.25} & 1.5 & \multicolumn{2}{|c|}{1.75} & 1.25 & \multicolumn{2}{|l|}{1.5} & 1.75 \\
\hline & $f_{e}(\%)$ & \multicolumn{2}{|c|}{5.08} & 3.56 & \multicolumn{2}{|c|}{2.58} & 6.04 & \multicolumn{2}{|l|}{4.30} & 3.19 \\
\hline
\end{tabular}

Table 3: Test matrix for the microstructures with saturated grain boundaries.

The matrix behavior parameters are those specified in section 5.1.

\subsubsection{Loading parameters}

Each microstructure is submitted to three distinct loading conditions with imposed macroscopic stress direction:

- a purely hydrostatic traction,

- a purely deviatoric axisymmetric loading $\left(\Sigma_{33}=-2 \Sigma_{11}=-2 \Sigma_{22}, \Sigma_{i j, i \neq j}=0\right)$,

- an intermediary axisymmetric sollicitation with a high (but finite) triaxiality ratio $\left(\Sigma_{m} / \Sigma_{e q}=4, \Sigma_{33}=1.27 \Sigma_{11}=1.27 \Sigma_{22}, \Sigma_{i j, i \neq j}=0, \Sigma_{m}\right.$ and $\Sigma_{e q}$ being the hydrostatic and equivalent parts of $\boldsymbol{\Sigma}$ ). 


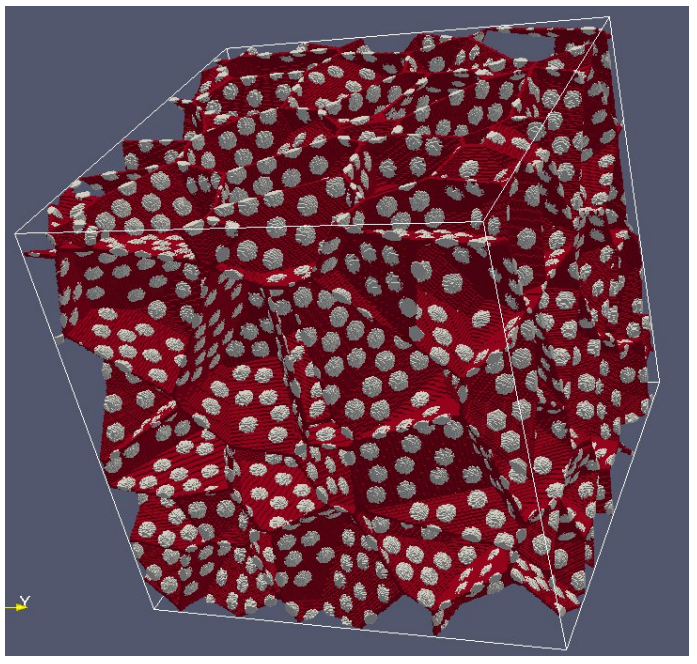

a) $r_{s} / r_{e}=1.25$

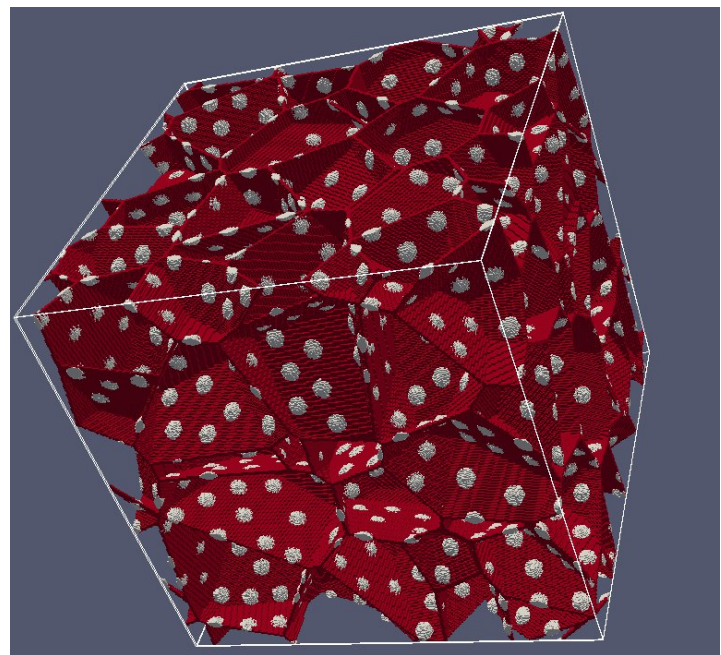

b) $r_{s} / r_{e}=1.75$

Figure 5: Examples of unit cells used in the FFT simulations: microstructures of a polycrystal with intergranular bubbles saturating the grain boundaries. Effect of two distinct minimal spacings between the bubbles. Red : grain boundaries. White : bubbles.

Moreover, one particular microstructure (21 grains, saturated boundaries, $r_{e} / r_{g}=0.2$, $\left.r_{s} / r_{e}=1.5\right)$ is tested under 16 different loading directions to estimate the overall shape for the effective plastic flow surface. This is also useful to assess the role of the third invariant of $\boldsymbol{\Sigma}$ on the effective plastic flow surface.

\subsubsection{Representativeness}

The representativeness of a volume element is still an open matter especially for non linear materials. The very first property of a representative volume element is its stationarity. For finite volumes, such as the ones corresponding to specific realizations of a unit-cell, one can only require that the deviation from stationarity should be less than a given threshold. In fact, this definition is strongly related to the quantity of interest. In the present study, the effective yield stress is the quantity of interest but the elastic properties are not. The stationarity can be controlled by drawing different polycrystals with the same intergranular parameters and by comparing their effective yield stresses.

Secondly, since the voided materials in this study are made of an isotropic matrix contaning voids located on untextured grain boundaries, macroscopic isotropy is a requirement for which the results should be checked. As explained in Vincent et al. (2014b), there are several possible definitions of the isotropy for a volume element. The isotropy of a volume element can be evaluated either by studying geometrical arrangement of the phases or by studying the mechanical response under the application of mechanical loadings for given 
constitutive relations of the phases. The first method can be called "geometrical isotropy". It can be assessed by considering the covariograms of the phases, as proposed by Kanit et al. (2003). The second method can be called "mechanical isotropy". A measure of deviation from mechanical isotropy was introduced by Vincent et al. (2014b). This measure is not recalled here and the reader is refered to Vincent et al. (2014b) to get more informations about it. The Tables B.5 and B.6 in Appendix B give the different isotropy deviations calculated after full-field simulations by means of this measure. As expected, different configurations may present significant deviations from the mechanical isotropy. However, it is worth noting that, for a given configuration, the deviation from the mechanical isotropy also depends on the loading direction : the deviation is important in the purely hydrostatic case (9 to $32 \%$ ), moderate in the case with a triaxiality ratio of 4 (2 to $15 \%$ ), and low in the purely deviatoric case (0 to $5 \%$ ). This evolution with the loading direction was already observed in Vincent et al. (2014b). As in (Vincent et al., 2014b), when averages of several configurations are taken, the deviation from isotropy is small: the line of the Table B.5 mentioning the word "mean" corresponds to the average obtained on three different realizations. As well, the deviation from isotropy for saturated grain boundaries are significantly lower in the case of a 80 grains volume than for a 21 grains volume.

The variations of the mechanical responses between different realizations of polycrystals with the same parameters for the bubbles is evaluated through three realizations for two sets of parameters (21 grains, saturated grain boundaries, $r_{e} / r_{g}=0.1, r_{s} / r_{e}=1.25$, and 21 grains, saturated grain boundaries, $\left.r_{e} / r_{g}=0.2, r_{s} / r_{e}=1.5\right)$. The greatest difference between the effective yield stresses of two realizations with the same set of microstructural parameters is less than $1 \%$ of the mean calculated effective yield stress. Therefore, despite the rather high anisotropy, it appears that only one realization with 21 grains is sufficient to investigate the effective plastic flow surface.

\subsection{Numerical results}

\subsubsection{Purely hydrostatic loading}

Figure 6 shows the effective hydrostatic stress obtained by the full-field simulations as a function of the volume fraction $f_{e}$. As expected, the bubbles have a softening effect on the effective yield surface (the hydrostatic yield stress decreases when the porosity $f_{e}$ increases). Moreover, a significant effect of the relative size of the bubbles is identified. It is recalled here that it is a relative size, keeping in mind that the mean size of the grains has been fixed. For example, for a given volume fraction $f_{e} \approx 2 \%$, dividing by two the bubble radius may lead to a $20 \%$ supplementary decrease of the yield stress. The results plotted in Figure 6 suggest that, for a given volume fraction $f_{e}$, the effective hydrostatic stress reduces when decreasing the relative size of the bubbles.

\subsubsection{Purely deviatoric loading}

Figure 7 shows the effective equivalent stress obtained by the full-field simulations as a function of the volume fraction $\left(f_{e}\right)$ for purely deviatoric axisymmetric loading. As for 


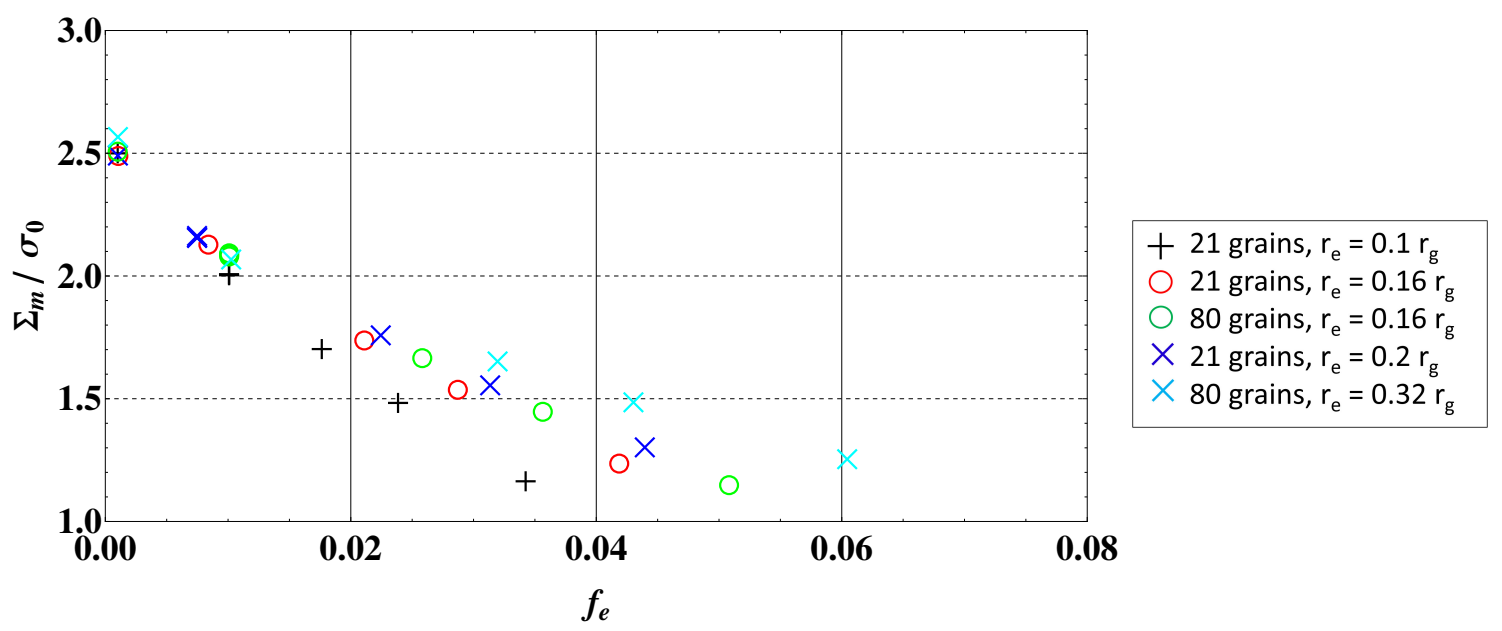

Figure 6: Effective hydrostatic stresses obtained by full-field simulations for a purely hydrostatic loading as a function of the volume fraction $f_{e}$ for different relative sizes of the bubbles $\left(r_{e} / r_{g}\right)$.

the purely hydrostatic case, the intergranular cavities lead to a softening of the material. The relative size of the bubbles has a non neglectable effect. Nevertheless, this effect is less important compared to the case of a purely hydrostatic loading. A factor of two on the bubble radius from $1 \mu \mathrm{m}$ to $2 \mu \mathrm{m}$ for a volume fraction of $3.5 \%$ leads to only $4 \%$ supplementary decrease of the yield stress.

\subsubsection{Intermediate loading}

Figure 8 shows the effective hydrostatic stress obtained by the full-field simulations as a function of the volume fraction $f_{e}$, for a high but finite loading triaxiality ratio $\left(\Sigma_{m} / \Sigma_{e q}=\right.$ $\left.4, \Sigma_{33}=1.27 \Sigma_{11}=1.27 \Sigma_{22}, \Sigma_{i j, i \neq j}=0\right)$. As for the purely hydrostatic and purely deviatoric loadings, the points can not be connected in a single curve. Therefore the relative size of the bubbles can not be neglected. A factor of two on the bubble radius may lead to a $20 \%$ variation of the effective hydrostatic stress, as in the case of the purely hydrostatic loading. As expected, the same evolution of the effective stress with the relative size of the bubbles appears than for the purely hydrostatic loading and the purely deviatoric loading.

\subsubsection{Conclusions}

From the numerical full-field simulations, it can be concluded that:

- the intergranular bubbles have a softening effect on the material;

- the relative size of the bubbles has a significant influence on the effective plastic flow 


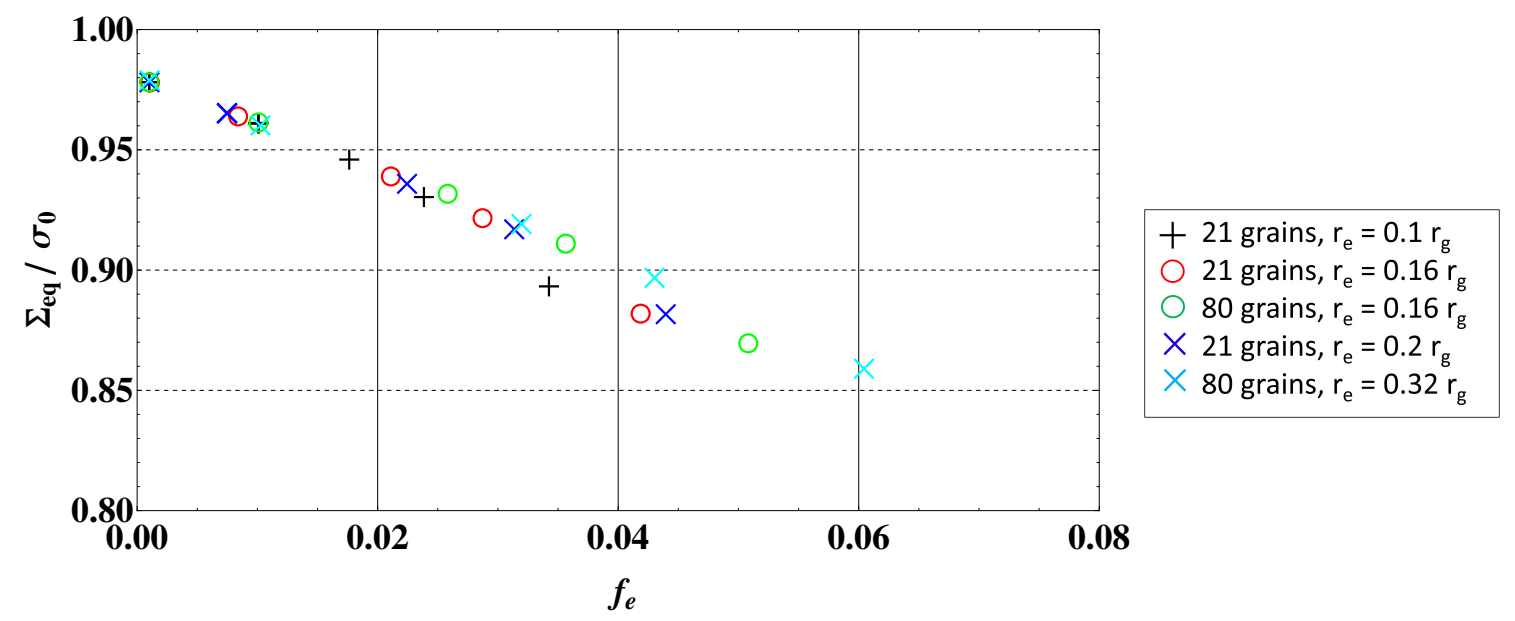

Figure 7: Effective yield stresses obtained by full-field simulations for a loading with imposed stress direction $\left(\Sigma_{33}=-2 \Sigma_{11}=-2 \Sigma_{22}, \Sigma_{i j, i \neq j}=0\right)$ as a function of the volume fraction $f_{e}$ for different relative sizes of the bubbles $\left(r_{e} / r_{g}\right)$.

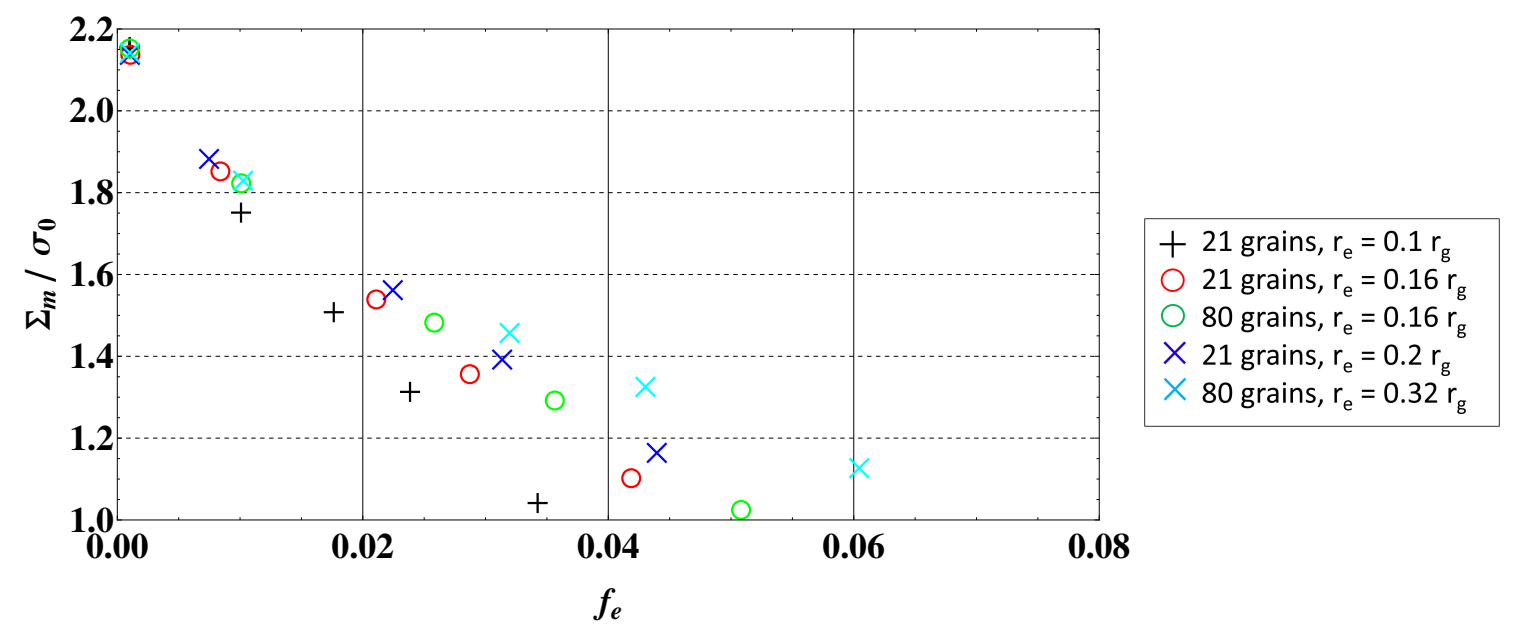

Figure 8: Effective hydrostatic stresses obtained by full-field simulations for a loading with imposed stress direction $\left(\Sigma_{33}=1.27 \Sigma_{11}=1.27 \Sigma_{22}, \Sigma_{i j, i \neq j}=0, \Sigma_{m} / \Sigma_{e q}=4\right)$ as a function of the volume fraction $f_{e}$ for different relative sizes of the bubbles $\left(r_{e} / r_{g}\right)$.

stress for a purely hydrostatic stress loading (for a given volume fraction of bubbles, the flow stress decreases when the relative size decreases).

- the relative size of the bubbles has a less important influence on the effective plastic 
flow stress for a purely deviatoric axisymmetric stress loading (for a given volume fraction of bubbles, the flow stress also decreases when the relative size decreases).

Again, one should keep in mind that the mean size of the grains is fixed, so that the sizes of the bubbles introduced here are in fact relative sizes.

These observations are in good agreement with those of Bilger et al. (2005) who have carried 3D simulations of porous media composed of a perfectly plastic matrix weakened by stress-free voids, with random position of the voids or with connected or disconnected clusters of voids. A simulated clustered microstructure with connected voids clusters is obtained from a microstructure with randomly distributed voids by removing voids in large disconnected spherical zones. Therefore distributing the void along grain boundaries is close to distributing them into connected clusters. The simulations exhibited a strong effect of connected cluster (compared to random distribution) on the yield stress during an hydrostatic loading but almost no effect on purely deviatoric loading as in the present study.

\subsection{Local plastic strain field}

Figures 9 and 10 show the typical aspect of the plastic strain field inside the microstructure for a purely hydrostatic loading and an axisymmetric purely deviatoric loading, respectively. For the purely hydrostatic loading test, the plasticity is located along the grain boundaries. For the purely deviatoric test, the cumulated plastic strain is high in some grain boundaries, but some plasticity also develops inside the grains. Such results may explain the difference observed in the influence of the relative size of the bubbles with the loading direction.

Figure 11 shows the difference between the plasticity localizations of two purely deviatoric stress loadings with different third invariants on the same microstructure. In the first case, the solicitation is $\left(\Sigma_{33}=-2 \Sigma_{11}=-2 \Sigma_{22}, \Sigma_{i j, i \neq j}=0\right)$ close to a uniaxial compression along $(\mathrm{Oz})$ axis. The plasticity is therefore activated in the weakest plans having a $45^{\circ}$ angle with (Oz) axis. In this case, they correspond to some grain boundaries. In the second case, the solicitation is pure shear along $\Sigma_{13}$. The plasticity is therefore activated in the weakest plans having a $45^{\circ}$ with the axis $(\mathrm{O},(1,0,1))$. Thus it corresponds to plans orthogonal to $(\mathrm{Oz})$ or $(\mathrm{Ox})$. Surprisingly, only one plan is activated in the whole volume instead of different pieces of plans as in the first case: it shows that this specific microstructure under this specific loading has a weakest plan and that it is not fully isotropic with respect to the local cumulated plastic strain field. It illustrates the fact that the microstructure can be isotropic with respect to the effective plastic flow surface but anisotropic for local fields such as the cumulated plastic strain.

\section{Identification of a simplified analytical model}

\subsection{Analytical model}

Vincent et al. (2014a,b) have derived a simplified analytical model for a biporous mate- 

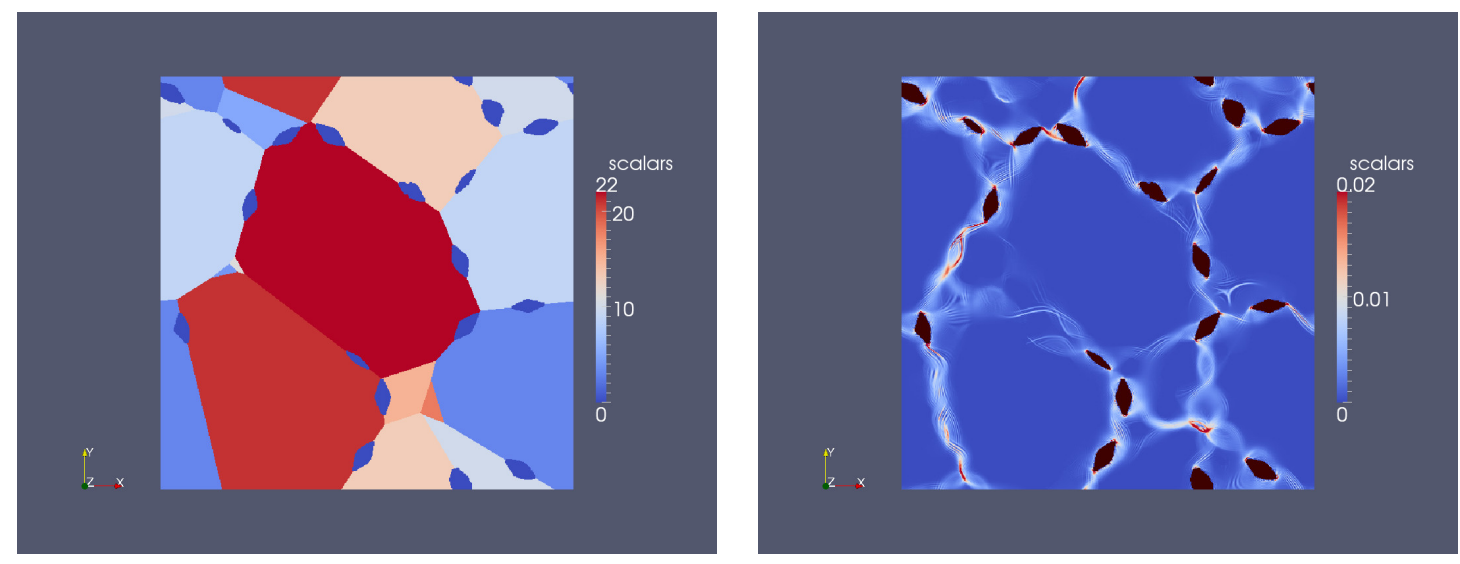

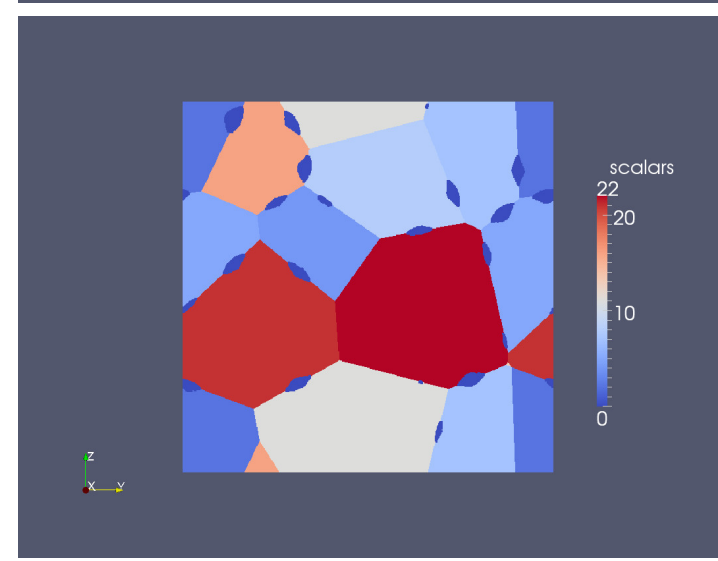

a) microstructure

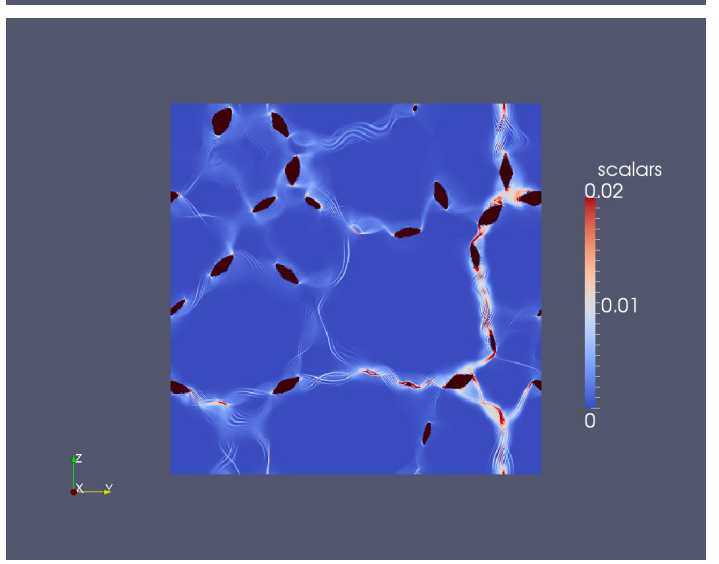

b) cumulated plastic strain

Figure 9: Two slices of the microstructure with 21 grains, saturated grain boundaries, $r_{e} / r_{g}=0.2, r_{s} / r_{e}=$ 1.5. Cumulated plastic strain fields for a purely hydrostatic overall stress.

rial, with small intragranular spherical bubbles and bigger ellipsoidal intergranular bubbles. An approximation of the effective plastic flow surface was identified. It is similar in the form to a Gurson, or GTN, model. It reads as:

$$
\frac{1}{\beta}\left(\frac{\Sigma_{e q}}{\sigma_{0}}\right)^{2}+\frac{1}{\alpha} \cosh \left(\frac{3}{2}\left(\frac{\Sigma_{m}}{\sigma_{0}}-\frac{\bar{\Sigma}_{m}^{+}+\bar{\Sigma}_{m}^{-}}{2 \sigma_{0}}\right)\right)-1=0,
$$

where $\Sigma_{m}$ is the macroscopic hydrostatic stress and $\Sigma_{e q}$ is the Von Mises stress. $\bar{\Sigma}_{m}^{+}$and $\bar{\Sigma}_{m}^{-}$ are the flow stresses of the material under hydrostatic stress (corresponding respectively to a contraction and a dilatation of the material). $\alpha$ and $\beta$ are the two scalar functions: 

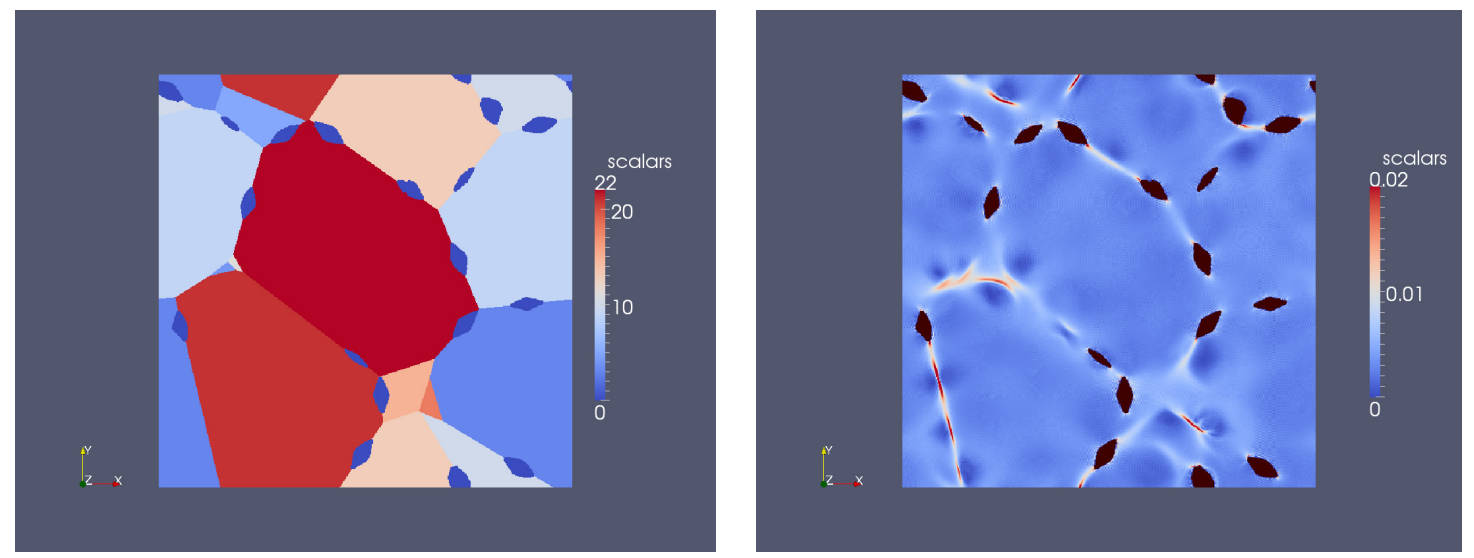

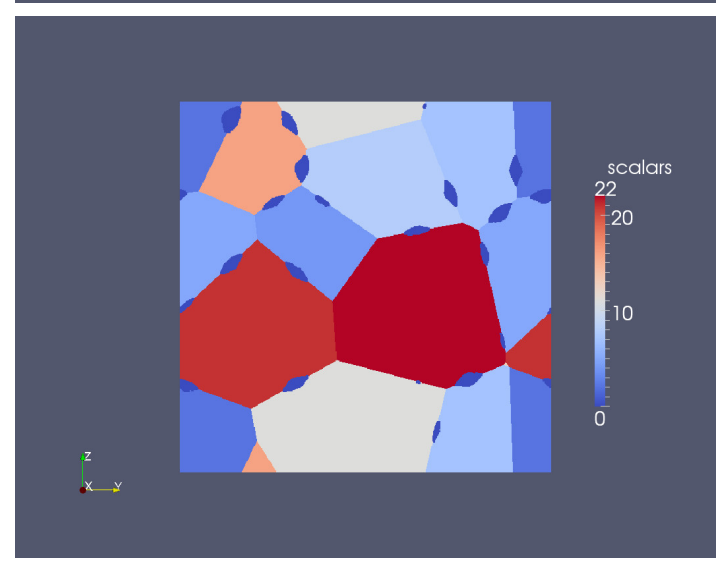

a) microstructure

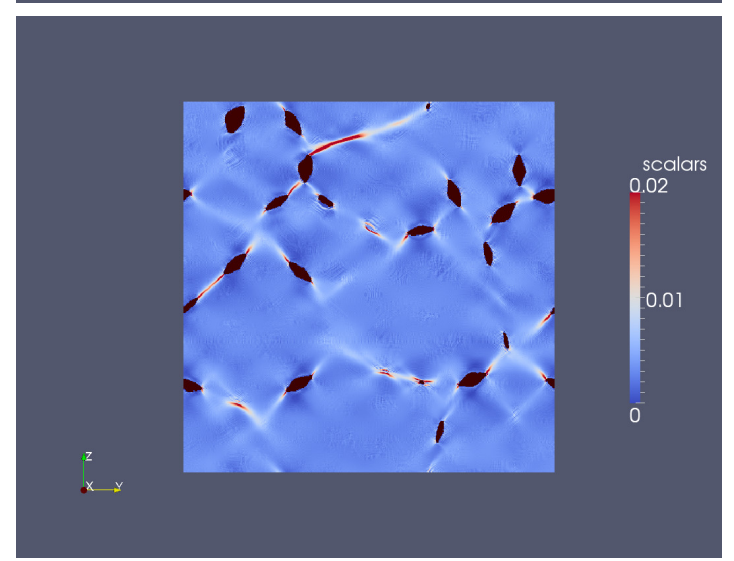

b) cumulated plastic strain

Figure 10: Two slices of the microstructure with 21 grains, saturated grain boundaries, $r_{e} / r_{g}=0.2$, $r_{s} / r_{e}=1.5$. Cumulated plastic strain fields for a purely deviatoric overall stress $\left(\Sigma_{33}=-2 \Sigma_{11}=-2 \Sigma_{22}\right.$ and $\left.\Sigma_{12}=\Sigma_{13}=\Sigma_{23}=0\right)$.

$$
\begin{aligned}
& \alpha=\cosh \left(\frac{3}{4}\left(\frac{\bar{\Sigma}_{m}^{+}-\bar{\Sigma}_{m}^{-}}{\sigma_{0}}\right)\right), \\
& \beta=\quad\left(\frac{\bar{\Sigma}_{e q}}{\sigma_{0}}\right)^{2} \frac{\alpha}{\alpha-1},
\end{aligned}
$$

where $\bar{\Sigma}_{e q}$ is the flow stress of the material under purely deviatoric strain-rate. $\bar{\Sigma}_{e q}, \bar{\Sigma}_{m}^{+}$ and $\bar{\Sigma}_{m}^{-}$depend on the shape and volume fractions of the two populations of voids and on the internal pressures, $P_{b}$ of the intragranular cavities, $P_{e}$ of the intergranular cavities. Thus, the proposed criterion is a generalized Gurson criterion passing through the three 

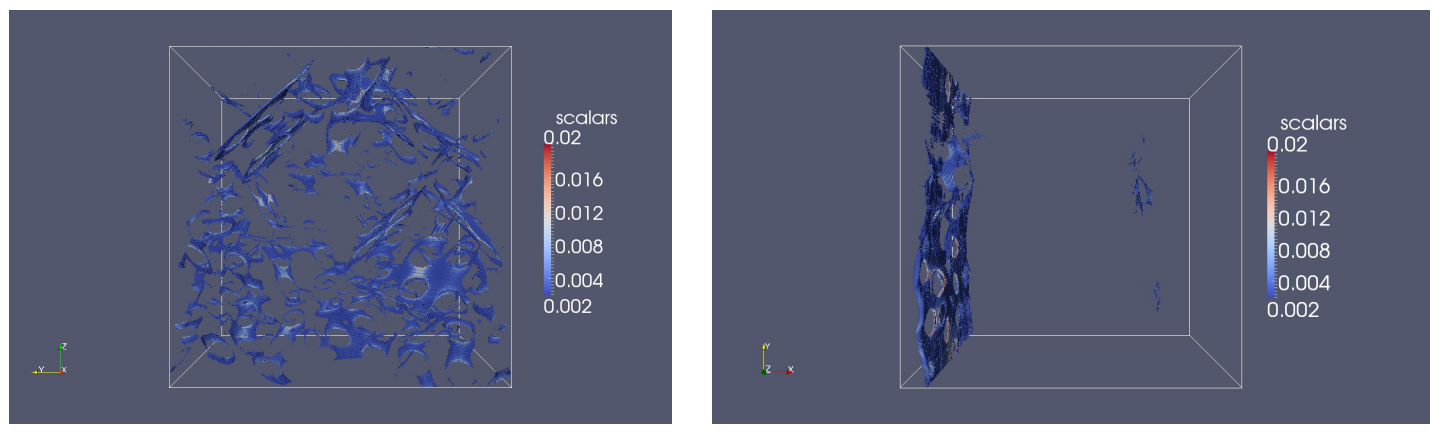

$$
\boldsymbol{\Sigma}=\left(\begin{array}{ccc}
S & 0 & 0 \\
0 & S & 0 \\
0 & 0 & -2 S
\end{array}\right) \quad \boldsymbol{\Sigma}=\left(\begin{array}{ccc}
0 & 0 & S \\
0 & 0 & 0 \\
S & 0 & 0
\end{array}\right)
$$

Figure 11: Cumulated plastic strain fields for two different purely deviatoric tests on the same microstructure (21 grains with saturated grain boundaries, $r_{e} / r_{g}=0.2, r_{s} / r_{e}=1.5$ ).

specific points $\bar{\Sigma}_{e q}, \bar{\Sigma}_{m}^{+}$and $\bar{\Sigma}_{m}^{-}$. The identification method is fully described in (Vincent et al., 2014a,b).

A comparison with numerical results obtained by full-field calculations with the FFT method was performed by Vincent et al. (2014b). It has shown the necessity to use a corrected volume fraction in the analytical model to achieve a good match with the numerical experiments. The correction depends on the aspect ratio $w_{e}$ (of the equivalent ellipsoidal cavities, see Appendix A) and the volume fraction, $f_{e}$, of the intergranular cavities. Due to the specific geometries considered here (elongated bubbles on grain boundaries), a new correction has to be identified.

\subsection{Identification of the corrected volume fraction}

Let us define the corrected volume fraction $f^{*}$ as the porosity to be considered in the analytical model instead of the true porosity $f_{e}$, in order to get, with the model, the overall stress obtained by numerical simulation. Figure 12 shows the existence of two separated domains, whatever the loading direction (purely hydrostatic or deviatoric), with a transition zone around $f_{e}=0.01$. The first domain corresponds to low volume fractions. In that domain, the relative size of the bubbles has a small influence on the effective yield stress, which depends mainly on the volume fraction of the bubbles. On the contrary, for high intergranular bubble volume fractions, both the volume fraction and the relative size of the bubbles play a role on the effective yield stress and it is more pronounced in the purely hydrostatic case than in the purely deviatoric case.

Thus, it appears that it depends on the loading direction. But, as only three specific values have to be identified for the analytical model $\left(\bar{\Sigma}_{e q}, \bar{\Sigma}_{m}^{+}\right.$, and $\left.\bar{\Sigma}_{m}^{-}\right)$, only two corrections are required. The first one, denoted by $f_{h}^{*}$, corresponds to the purely hydrostatic loading 
and is relative to $\bar{\Sigma}_{m}^{-}$and $\bar{\Sigma}_{m}^{+}$. The second one, denoted $f_{d}^{*}$, corresponds to the purely deviatoric loading and is relative to $\bar{\Sigma}_{e q}$. These functions are searched in the following form:

$$
f_{h, d}^{*}=g_{h, d}\left(f_{e}\right)+h_{h, d}\left(f_{e}, r_{e} / r_{g}\right) .
$$

The functions $g_{d}$ and $g_{h}$ are identified by the least squares method from Figure 12 (brown curve) by considering only the points in the region where $f_{e} \leq 0.01$. The functions $h_{h}$ and $h_{d}$ are supposed to be power law functions with respect to two variables, the volume fraction of the intergranular bubbles $f_{e}$ and the normalized size of the bubbles $\left(r_{e} / r_{g}\right)$. A straightforward analysis using the least squares method on the functions $f_{h, d}^{*}-g_{h, d}$ leads to the following empirical relations:

$$
\begin{aligned}
& f_{d}^{*}=1.9 f_{e}+25\left(\frac{r_{g}}{r_{e}}\right)^{2} f_{e}^{7 / 2} \\
& f_{h}^{*}=0.5 f_{e}^{3 / 4}+4.3\left(\frac{r_{g}}{r_{e}}\right)^{2} f_{e}^{5 / 2} .
\end{aligned}
$$

The calculated (full-field simulations) and estimated (analytical model) effective yield stresses with the identified $f_{h, d}^{*}$ are plotted in Figure 13. As a result, the simple analytical expression of the effective flow surface of Vincent et al. (2014a,b) is proposed (equations (16), (17), (18)) with corrected volume fractions: $\bar{\Sigma}_{m}^{-}$and $\bar{\Sigma}_{m}^{+}$are evaluated with $f_{h}^{*}$ instead of the true porosity $f_{e}$, and $\bar{\Sigma}_{e q}$ is evaluated with $f_{d}^{*}$ instead of the true porosity $f_{e}$.

\subsection{Validation and discussion}

\subsubsection{Intragranular porosity}

The proposed corrected bubbles volume fractions $f_{d}^{\star}$ and $f_{h}^{\star}$ are independent of the local intragranular porosity $f_{b}$ which here is a parameter of the GTN model. Since $f_{b}$ and $f_{e}$ are both void volume fractions, it is worth verifying that the expressions of $f_{d}^{\star}$ and $f_{h}^{\star}$ are valid for different values of $f_{b}$.

In Figure 14, the calculated effective yield stresses are compared with those predicted by the analytical model with corrected bubbles volume fraction for two different intragranular porosities: $f_{b}=6 \%$ and of $f_{b}=0.7 \%$. The agreement between the model predictions and the numerical simulations is good, for different microstructures and for both the purely deviatoric case $\left(\Sigma_{33}=-2 \Sigma_{11}=-2 \Sigma_{22}, \Sigma_{i j, i \neq j}=0\right)$ and the purely hydrostatic case.

\subsubsection{Effective plastic flow surface}

One specific microstructure (21 grains, saturated boundaries with $r_{e} / r_{g}=0.2, r_{s}=1.5$ $\left.r_{e}\right)$ is submitted to 16 different loadings. The differences between the effective yield stress of the full-field simulations and the analytical model are below $5 \%$, as it can be seen in Figure 15. Two types of loading are tested. Both are in the form: 

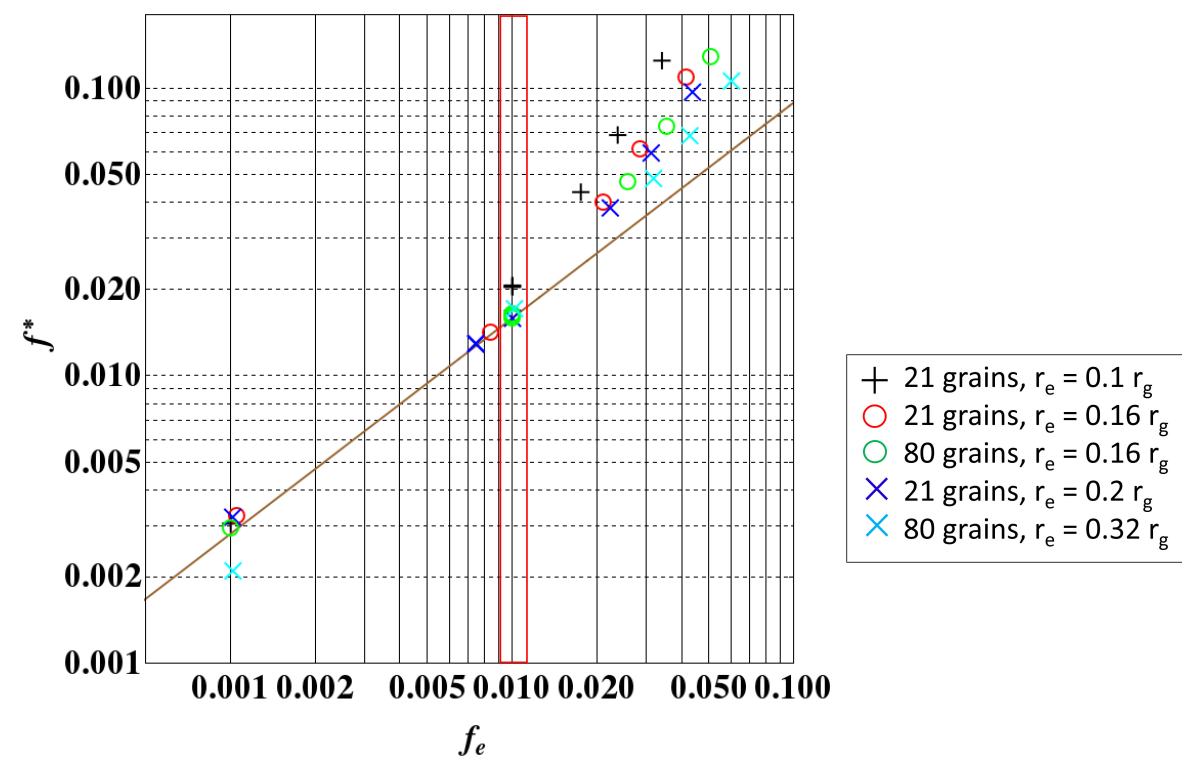

a) purely hydrostatic loading (brown line: $f^{*}=0.5 f_{e}^{3 / 4}$ )

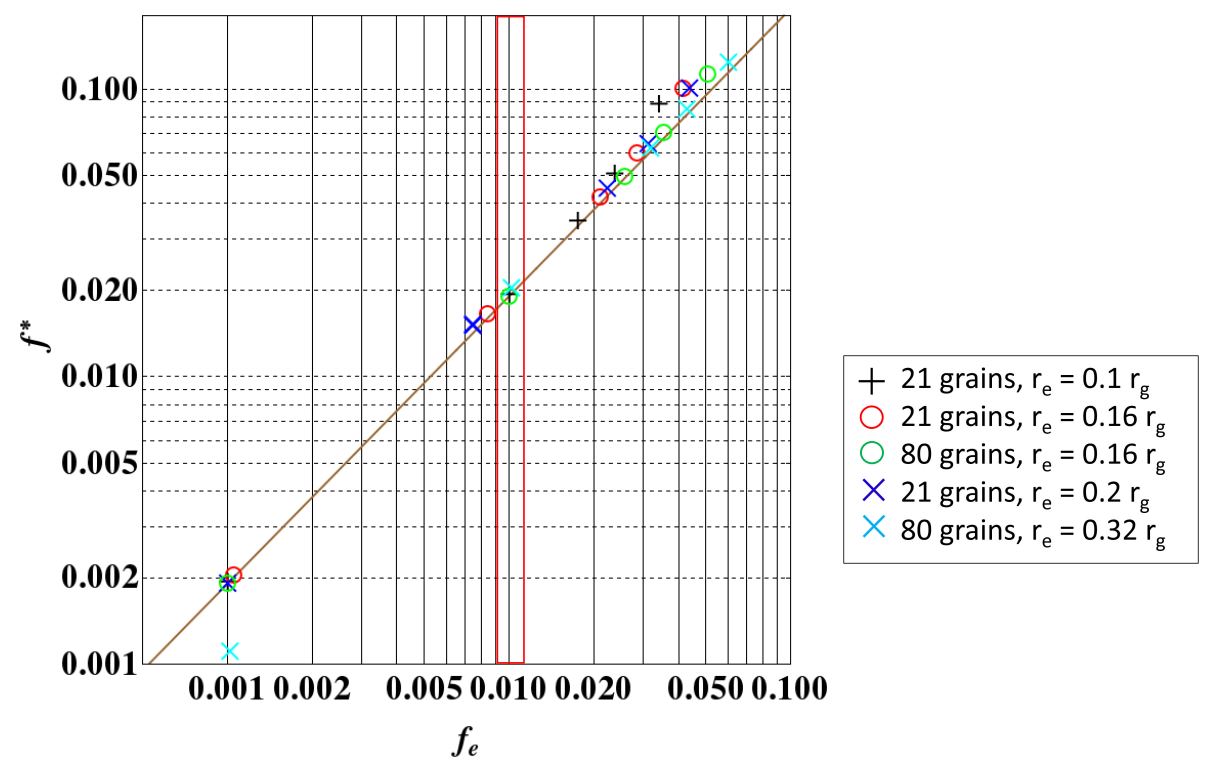

b) purely deviatoric loading (brown line: $f^{*}=1.9 f_{e}$ )

Figure 12: Identification of the corrected volume fraction $f^{*}$ (defined as the porosity to be considered in the analytical model instead of the true porosity $f_{e}$, in order to get, with the model, the overall stress obtained by numerical simulation). For low volume fractions $f_{e}$, the relative size of the bubbles has a small influence on the effective yield stress. For high volume fractions $f_{e}$, the relative size of the bubbles plays a role on the effective yield stress. The transition zone is framed in red. 


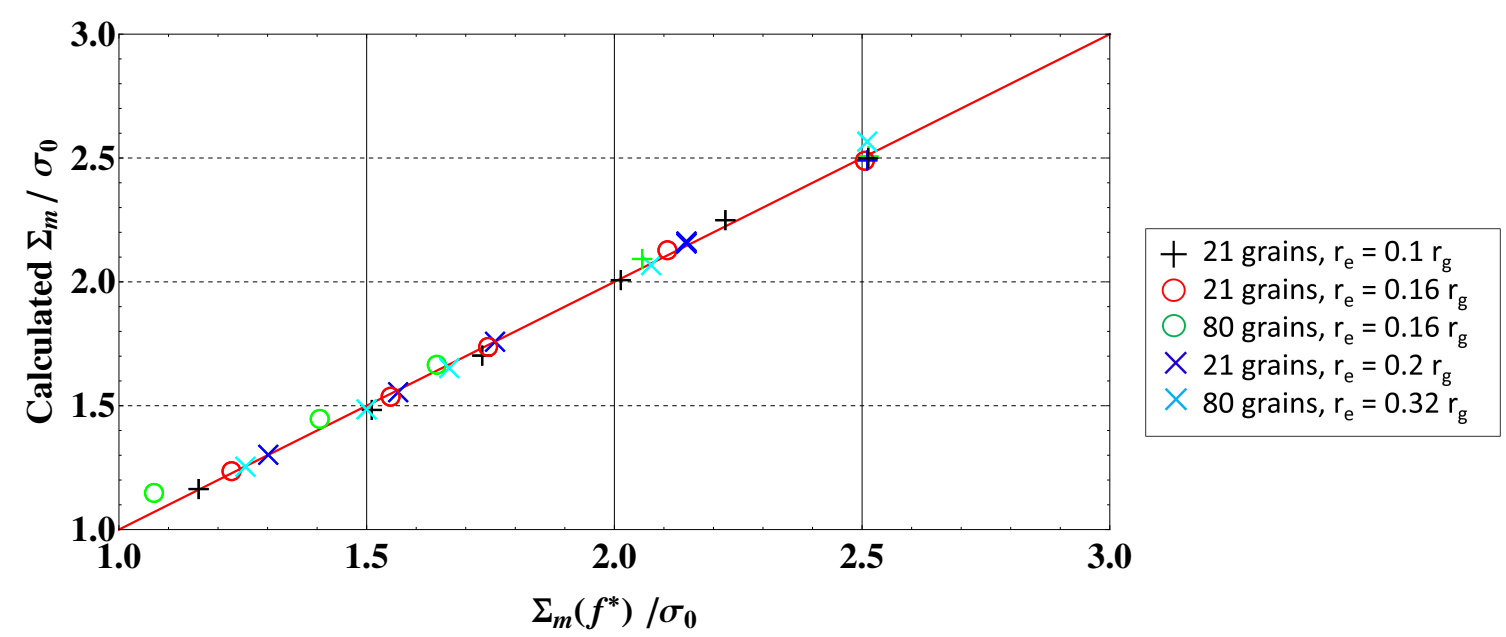

a) purely hydrostatic loading

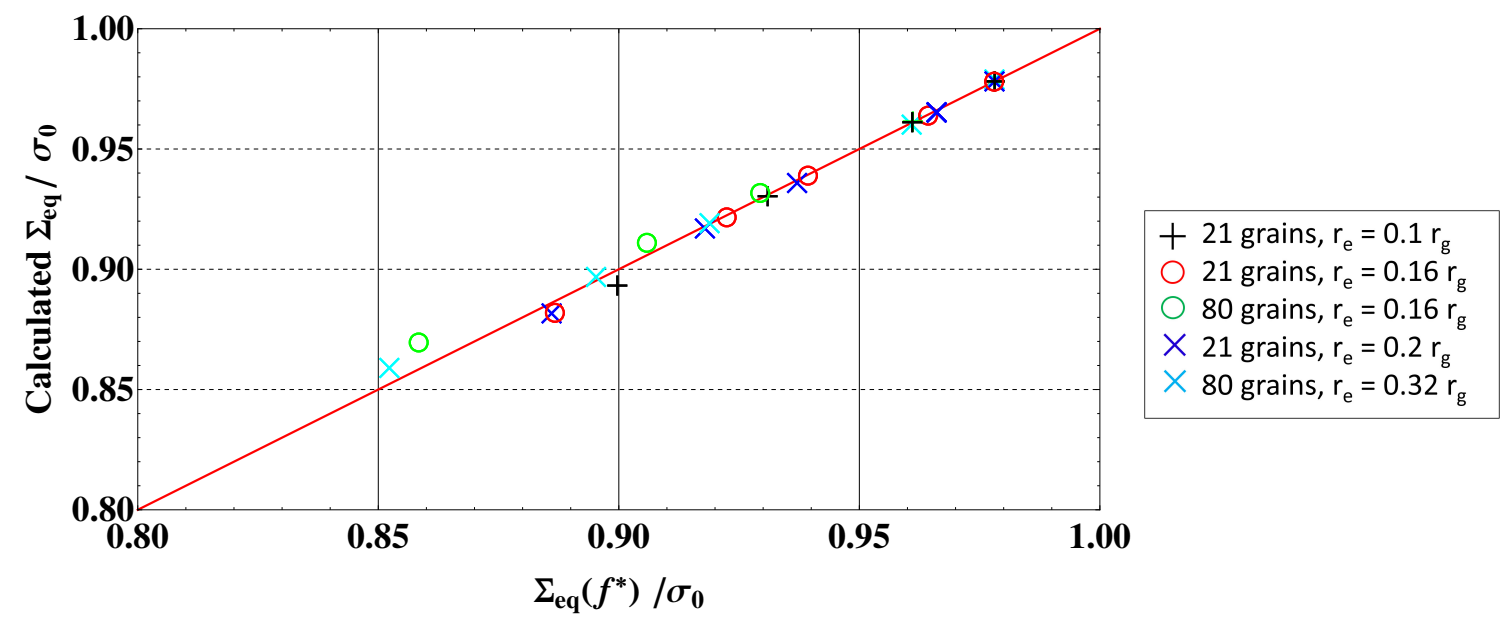

b) purely deviatoric loading

Figure 13: Estimated (analytical model) versus calculated (full-field simulations) effective yield stress.

$$
\boldsymbol{\Sigma}=\left(\begin{array}{ccc}
S & 0 & 0 \\
0 & S & 0 \\
0 & 0 & T
\end{array}\right)
$$

The first one corresponds to $|T|>|S|$ and the second one to $|T|<|S|$. Couples of stress directions can be made such that the directions of a couple have the same triaxility but different third invariants. Figure 15 shows that the results corresponding to $|T|>|S|$ have systematically a lower yield stress than the case $|S|>|T|$. It has been checked that this 


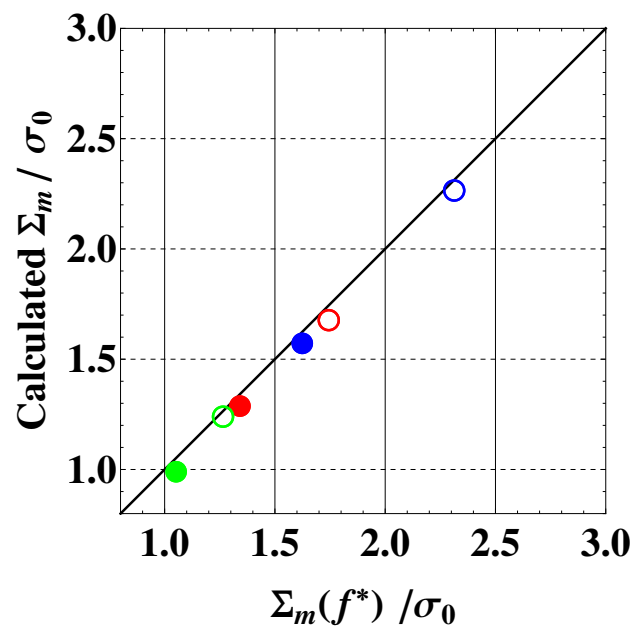

a) purely hydrostatic loading

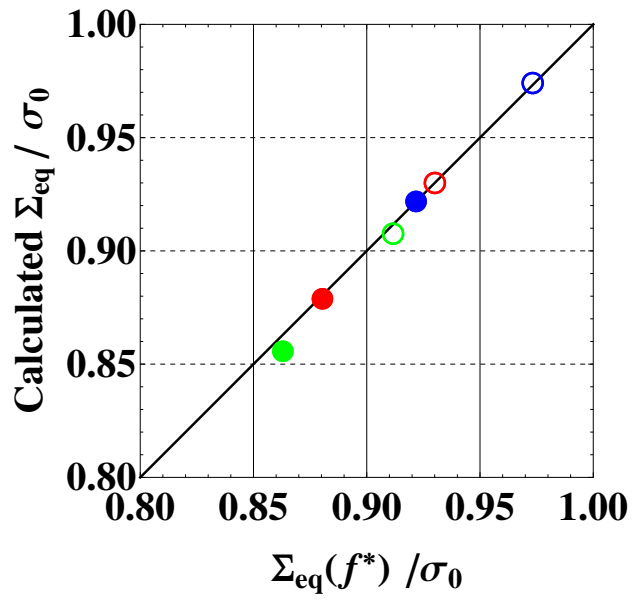

b) purely deviatoric loading

Figure 14: Numerical results (calculated values) for $f_{b}=0.7 \%$ (circles) and $f_{b}=6 \%$ (disks) compared to the analytical model. Three microstructures with 21 grains are tested: $i$. saturated grain boundaries, $r_{e} / r_{g}=0.2, r_{s} / r_{e}=1.5$ (red), ii. saturated grain boundaries, $r_{e} / r_{g}=0.1, r_{s} / r_{e}=1.25$ (green), iii. $f_{e}=$ $1 \%, r_{e} / r_{g}=0.1, r_{s} / r_{e}=1.75$ (blue).

result remains true for two other polycrystals with the same microstructural parameters. As a conclusion, the lower yield stress corresponding to the stress directions with $|T|>|S|$ is a third invariant effect and not an effect of a particular drawing of the polycrystal, as already exhibited by Julien et al. (2011); Cazacu et al. (2013); Vincent et al. (2014b).

\subsubsection{Saturated cavities}

In the case where the bubbles are pressurized (pressure $P_{b}$ inside the intragranular bubbles and $P_{e}$ inside the intergranular bubbles), Vincent et al. (2009a) have shown that the effective flow surface can be obtained from the problem with drained intragranular cavities $\left(\tilde{P}_{b}=0\right)$, provided that the pressure in the intergranular bubbles is set to $\tilde{P}_{e}=$ $P_{e}-P_{b}$. Equivalently, it can be obtained from the problem with drained intergranular cavities $\left(P_{e}^{*}=0\right)$, provided that the pressure in the intragranular bubbles is set equal to $P_{b}^{*}=P_{b}-P_{e}$. It was chosen to compare the analytical model with the corrected volume fraction $f^{*}$ in the case where $P_{b}=0$ and $P_{e} \neq 0$. Figure 16 shows a good agreement between the model and the simulations for a specific microstructure and different stress triaxiality ratios (the overall stresses are in the form (22)). Note that, for the full-field calculations, the intergranular bubble pressure $P_{e}$ increased linearly $\left(P_{e}(t)=P_{e 0} t\right)$ until it reached a prescribed value, which is then kept constant. Thus, the problem starts with an unloaded material. Otherwise, the pressure alone may load the material enough to reach the flow surface. 


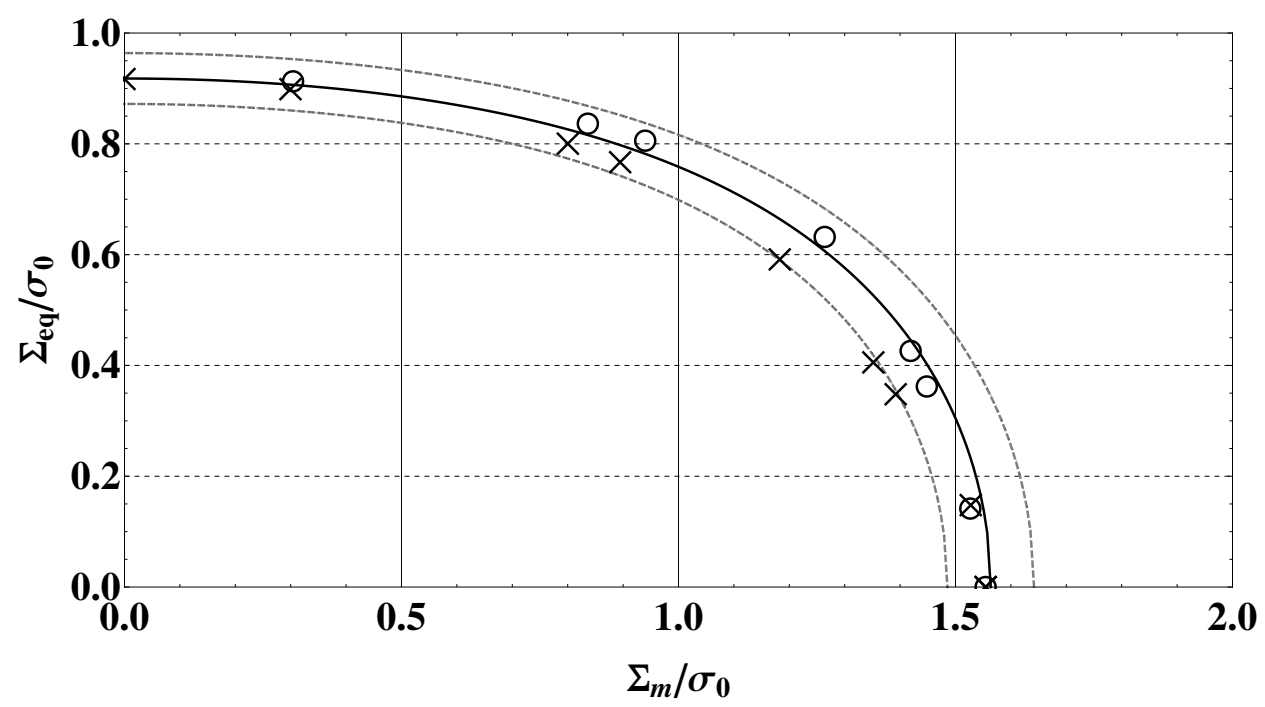

Figure 15: Numerical results (crosses: $|T|>|S|$, circles: $|S|>|T|$ ) compared to the analytical model (solid line) (microstructure: 21 grains, saturated grain boundaries, $r_{e} / r_{g}=0.2, r_{s} / r_{e}=1.5$ ). Dashed lines: estimate $\pm 5 \%$.

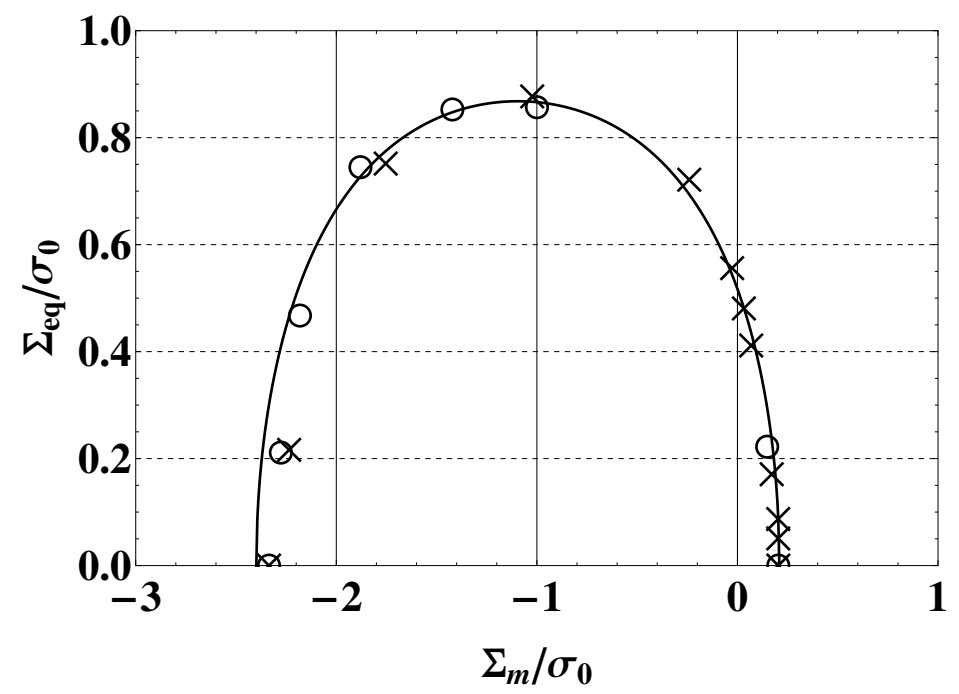

Figure 16: Numerical results (crosses: $|T|>|S|$, circles: $|S|>|T|$ ) compared to the analytical model (solid line) with $P_{e}=1.5 \sigma_{0}$ instead of 0 (microstructure: 21 grains, saturated grain boundaries, $r_{e} / r_{g}=0.2$, $\left.r_{s} / r_{e}=1.5\right)$. 


\subsubsection{Average dilatation-rate in the matrix}

The analytical model is based on an estimate of the average dilatation-rate in the matrix. In order to assess its validity, the strain-rate field obtained through the FFT simulations can be averaged over the matrix phase and compared to the estimate provided by the analytical model. As pointed out by Vincent et al. (2014b), the average dilatationrate in the matrix is crucial when dealing with the evolution of the void volume fractions. Indeed, the evolution of the porosity of the intergranular bubbles $f_{e}$ is governed by:

$$
\dot{f}_{e}=3\left(1-f_{e}\right)\left(\dot{E}_{m}-\left\langle\dot{\varepsilon}_{m}\right\rangle_{M}\right)
$$

where $\dot{E}_{m}$ is the hydrostatic overall strain rate and $\left\langle\dot{\varepsilon}_{m}\right\rangle_{M}$ is the average of the hydrostatic strain rate in the matrix. As a result, an accurate prediction for the evolution of $f_{e}$ needs an accurate estimate of $\left\langle\dot{\varepsilon}_{m}\right\rangle_{M}$. Similarly, the evolution law for the local intragranular void volume fraction $f_{b}$, which, rigorously, varies from point to point, writes:

$$
\dot{f}_{b}(\boldsymbol{x})=3\left(1-f_{b}(\boldsymbol{x})\right) \dot{\varepsilon}_{m}(\boldsymbol{x}) .
$$

This relation is averaged to give:

$$
\left\langle\dot{f}_{b}\right\rangle=3\left(1-f_{b}\right)\left\langle\dot{\varepsilon}_{m}\right\rangle_{M}
$$

Again, an accurate prediction for the evolution of $f_{b}$ needs an accurate estimate of $\left\langle\dot{\varepsilon}_{m}\right\rangle_{M}$.

The average of the hydrostatic strain rate in the matrix obtained by means of the model and the FFT simulations are compared on Figure 17 in the case of the purely hydrostatic loading (the case where the evolution of the porosities is supposed to be predominant). The computations are performed on the same microstructure as in 6.3.3. The case of the equivalent drained intragranular voids $\tilde{P}_{b}=0$ is considered and several values for $\tilde{P}_{e}$ are tested (Negative values of $\tilde{P}_{e}=P_{e}-P_{b}$ correspond to cases where $P_{b}$ is higher than $P_{e}$ ). A good agreement between FFT and model is observed, which confirms the validity of the model.

\subsubsection{Other topology of the grains}

In the present study, the geometry for the grains is based on a Poisson-Voronoi diagram. This choice is motivated by the recent study of Soulacroix (2014), who shows that this type of diagram is quite representative of the microstructure for polycrystals without particular characteristic. In order to remain general, a Voronoi tesselation is employed in the present study to generate the polycrystals. The potential effect of other topologies of the grains on the present results is difficult to estimate. Indeed, the application of the present method should be investigated for other types of tesselations, such as those of JohnsonMehl-Avrami-Kolmogorov and Laguerre. The curvy grain boundaries of such tesselations could lead to meshing problem for finite element calculation (non convexity problems), but 


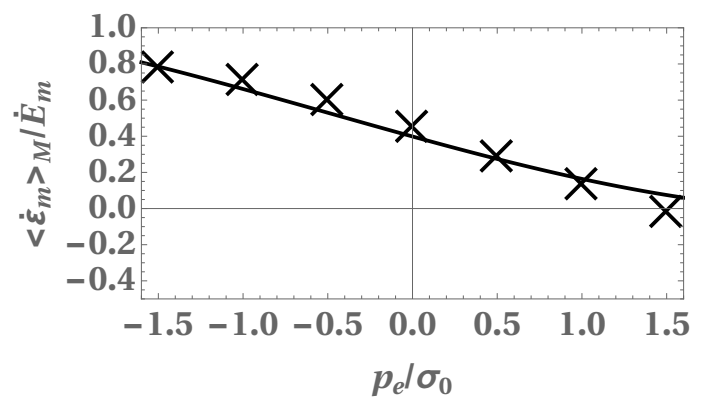

Figure 17: Average of the hydrostatic strain rate in the matrix (normalized by the hydrostatic overall strain rate) obtained by means of the model (line) and the FFT simulations (crosses) with respect to the intergranular internal pressure (normalized by the yield stress of the matrix without cavity).

present no difficulty for fast-Fourier-transforms method. As a preliminary study, 4 additional microstructures with a Johnson-Mehl-Avrami-Kolmogorov tesselation have been generated. Each microstructure contains 80 grains. A Johnson-Mehl-Avrami-Kolmogorov tesselation is obtained with a constant radial growth rate (1.0 voxel. $\mathrm{s}^{-1}$ in our case) as for Voronoi tesselation, but with different times at which each grain starts to grow (nucleation time). For each grain, this start-time consists in a value from 0 to a specific upper bound denoted by $t_{\max }$ (from a uniform distribution). The 4 microstructures considered here have the following upper bounds $t_{\max }$ for the time interval: $0,0.1 \mathrm{~s}, 0.25 \mathrm{~s}, 0.7 \mathrm{~s}$. Note that $t_{\max }=0$ corresponds to a Voronoi tesselation. The same location for the seeds (centers of the grains) is employed (from a uniform distribution). Nevertheless, some of the seeds did not correspond to a grain because their apparition time is too late. To obtain 80 grains each times, 80 seeds were drawn in the case $t=0.1 \mathrm{~s}, 96$ in the case $t=0.25 \mathrm{~s}$ and 318 in the case $\mathrm{t}=0.7 \mathrm{~s}$. Therefore the last case can be considered to have an almost completely different seeds than the others. The obtained polycrystals exhibit different distributions concerning the size of the grains. The 4 microstructures are shown on Figure 18. Histogram of the equivalent radius of grain $\left(r_{i}^{e q}\right)$ defined in equation 26) are plotted on Figure 19 for these 4 microstructures, showing that the Voronoi case corresponds to a narrower distribution for the size of the grains than the other cases. Then, intergranular bubbles are inserted on the grain boundaries with the following characteristics: $r_{e} / r_{g}=1.63 \mu m, r_{s} / r_{e}=1.5$, $f_{e}=2.97 \%, \theta=45^{\circ}$. The 4 resulting microstructures with intergranular voids are shown on Figure 20.

$$
r_{i}^{e q}=\left(\frac{3}{4 \pi V_{i}}\right)^{1 / 3}
$$

where $V_{i}$ denotes the number of voxels in the grain $i$. 


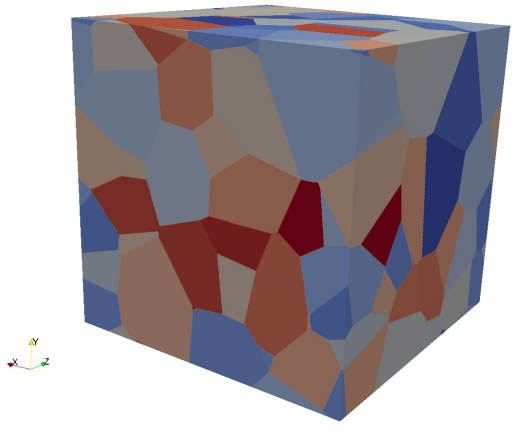

$t_{\max }=0$

Microstructure 1

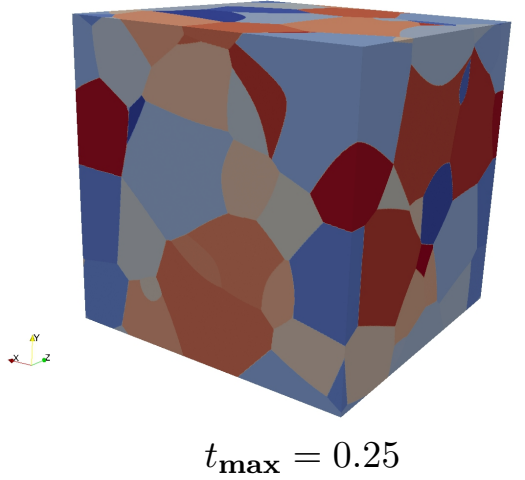

Microstructure 3

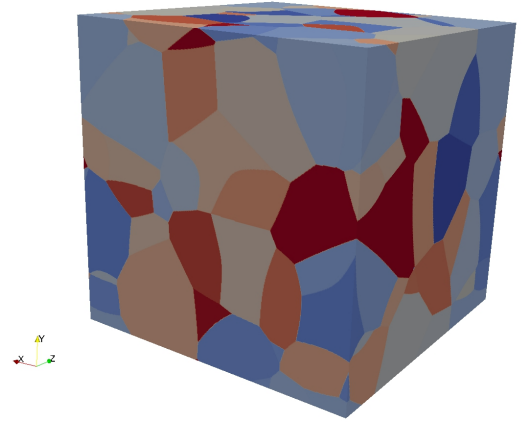

$t_{\max }=0.1$

Microstructure 2

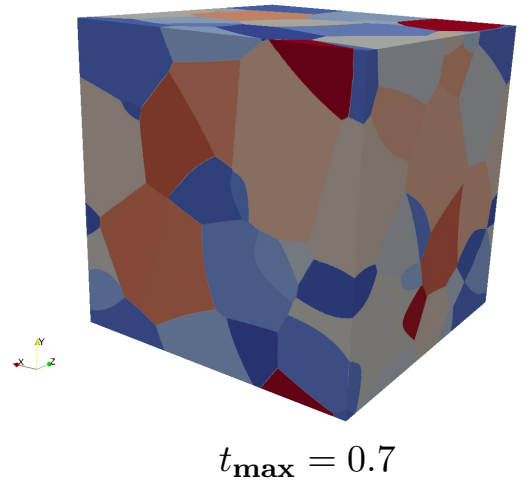

Microstructure 4

Figure 18: 4 microstructures generated from a Johnson-Mehl-Avrami-Kolmogorov tesselation. For each microstructure, the upper bound for the time interval for the nucleation of the grains is specified.

The 4 microstructures shown on Figure 20 are subjected to a purely hydrostatic, then a purely deviatoric loading. The overall yield stresses are reported on the Table 4 and compared to the analytical model. An excellent agreement between FFT and model is observed for the deviatoric loading an a good agreement is observed for the purely hydrostatic loading. For theses loadings, the microstructures with broader distribution of grain size appears to have a slightly lower effective yield stress. Nevertheless, this effect is of second order compared to the relative size of the voids compared to the grain size and compared to a random distribution of the voids (line "model with $f_{e}$ " on the Table 4). This preliminary study indicates that the model is still valid for some other topologies for the grains.

Some other interesting points are also exhibited : as in the case of a purely hydrostatic loading, the plasticity is located only around the voids and therefore along the grain boundaries, a larger grain leads to a larger plasticity free zone and thus to a higher plasticity 


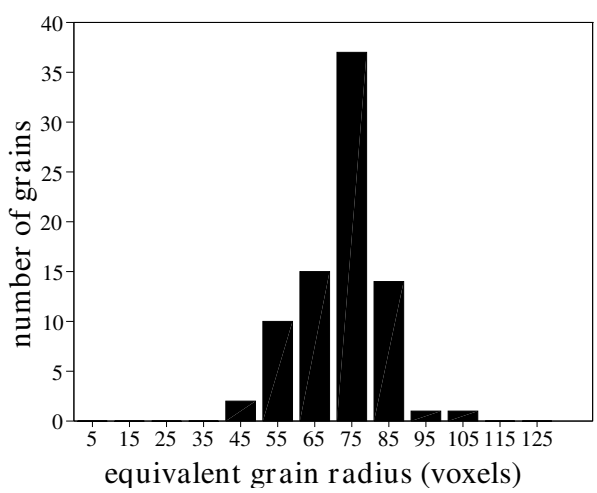

Microstructure 1

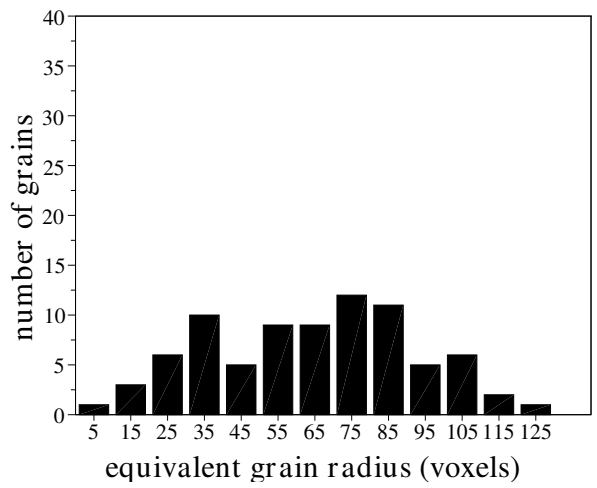

Microstructure 3

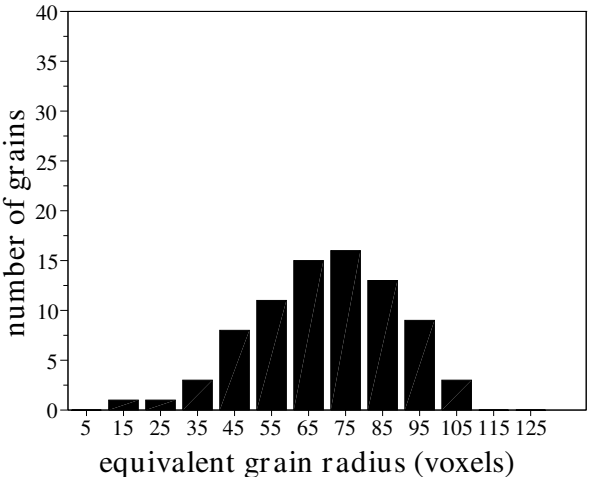

Microstructure 2

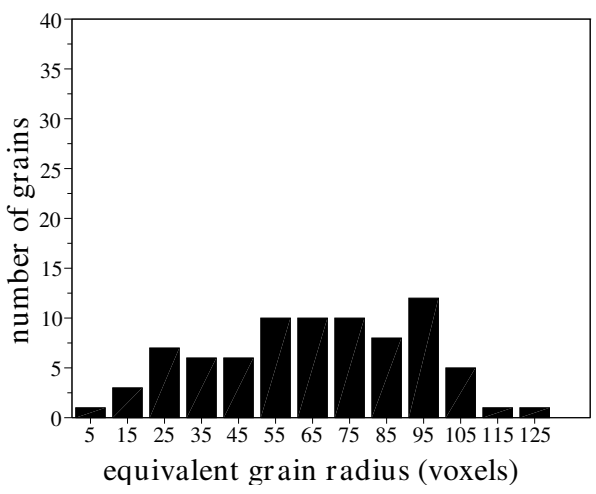

Microstructure 4

Figure 19: Histograms for the 4 microstructures generated from a Johnson-Mehl-Avrami-Kolmogorov tesselation.

along grain boundaries. Therefore, it is probable that the mean grain size as defined in the section 3 is not well adapted for broad distributions of grain sizes. An other definition which do not take into account the smallest grains would probably be better. However, to further validate the model and find a better definition of the mean grain size, the application of the present method should be investigated for a greater amount of different tesselations, together with other parameters for the voids (such as the porosity, the relative size, the location, and the internal pressure), in future work. 


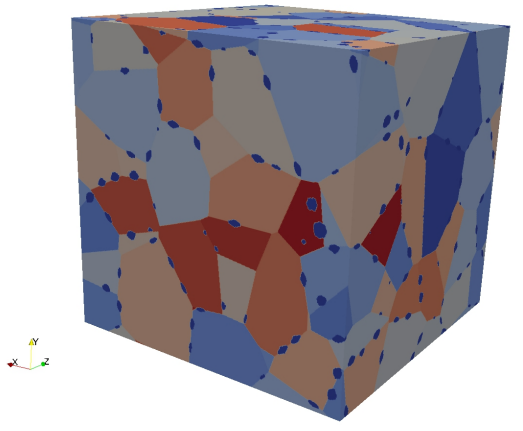

$t_{\max }=0$

Microstructure 1

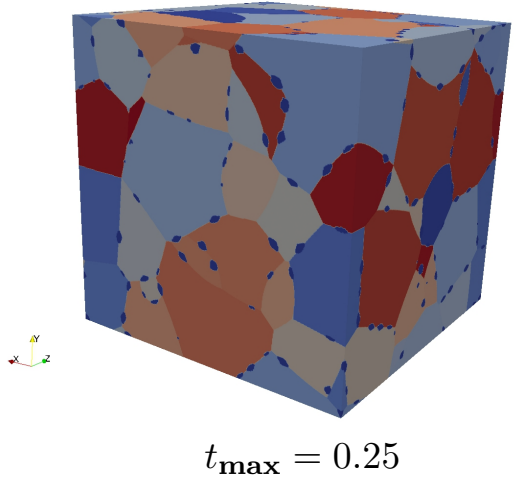

Microstructure 3

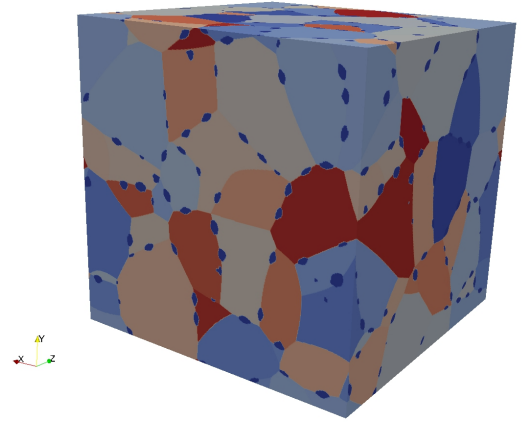

$t_{\max }=0.1$

Microstructure 2

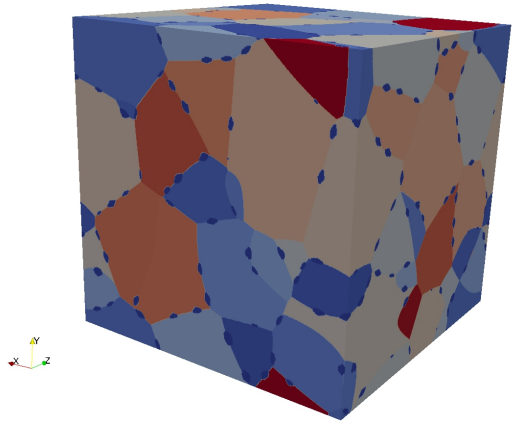

$t_{\max }=0.7$

Microstructure 4

Figure 20: Intergranular voids are put inside the 4 microstructures generated from a Johnson-MehlAvrami-Kolmogorov tesselation.

\begin{tabular}{|c|c|c|c|c|}
\hline Microstructure & 1 & 2 & 3 & 4 \\
\hline$\Sigma_{m} / \sigma_{0}$ FFT & 1.60 & 1.58 & 1.54 & 1.54 \\
\cline { 2 - 5 }$\Sigma_{m} / \sigma_{0}$ model with $f^{*}$ & \multicolumn{4}{|c|}{1.55} \\
\cline { 2 - 5 }$\Sigma_{m} / \sigma_{0}$ model with $f_{e}$ & \multicolumn{4}{|c|}{1.87} \\
\hline$\Sigma_{e q} / \sigma_{0}$ FFT & 0.924 & 0.924 & 0.924 & 0.924 \\
\cline { 2 - 3 }$\Sigma_{m} / \sigma_{0}$ model with $f^{*}$ & \multicolumn{4}{|c}{0.921} \\
\cline { 3 - 3 }$\Sigma_{m} / \sigma_{0}$ model with $f_{e}$ & \multicolumn{4}{|c|}{0.951} \\
\hline
\end{tabular}

Table 4: Overall yield stresses obtained for a purely hydrostatic, and a purely deviatoric loading, on the 4 microstructures generated from a Johnson-Mehl-Avrami-Kolmogorov tesselation.

\section{Conclusion}

The effect on the effective plastic flow surface of the relative size of intergranular lenticular cavities on the grain boundaries (relative size with respect to the mean grain size) 
in a biporous material with spherical intragranular cavities has been investigated through full-field numerical simulations with a FFT method. A GTN model was used to simulate the effect of the intragranular bubbles. The results have been compared to the analytical model of Vincent et al. (2014a). The main results are summarized below:

- The effect of the relative size of the intergranular bubbles on the effective plastic flow surface depends on the loading direction. It is larger for a purely hydrostatic overall stress direction than for a purely deviatoric axisymmetric overall stress direction. For a given volume fraction of the intergranular bubbles, the effective yield stress reduces when decreasing the relative size of the bubbles.

- The analytical model can be used with a correction of the intergranular bubble volume fraction to take into account the distribution of the intergranular bubbles along the grain boundaries. The model is predictive for a broad range of intergranular bubble relative sizes and volume fractions, and with pressurized intra- and/or inter-granular bubbles.

- This correction is split into two terms, which correspond to two distinct effects:

- an effect of volume fraction of intergranular bubbles independent of their relative size, which is predominant for a low volume fraction of intergranular bubbles,

- an effect of relative size and volume fraction of intergranular bubbles, which is predominant for a high volume fraction of intergranular bubbles.

\section{References}

Bhattacharya, K., Suquet, P., 2005. A model problem concerning recoverable strains of shape-memory polycrystals. Proc. R. Soc. A 461, 2797-2816.

Bilger, N., Auslender, F., Bornert, M., Michel, J.-C., H.Moulinec, Suquet, P., Zaoui, A., 2005. Effect of a nonuniform distribution of voids on the plastic response of voided materials: a computational and statistical analysis. International Journal of Solids ans Structures 42, 517-538.

Cazacu, O., Revil-Baudard, B., Lebensohn, R., Garajeu, M., 2013. On the combined effect of pressure and third invariant on yielding of porous solids with von mises matrix. J. Appl. Mech. 80 (6).

Dherbey, F., Louchet, F., Mocellin, A., Leclercq, S., 2002. Elevated temperature creep of polycrystalline uranium dioxide: from microscopic mechanisms to macroscopic behaviour. Acta Mater. 50 (6), 1495 - 1505.

Dubourg, R., Faure-Geors, H., Nicaise, G., Barrachin, M., 2005. Fission product release in the first two PHEBUS tests FPT0 and FPT1. Nucl. Eng. Des. 235, $2183-2208$. 
Eyre, D., Milton, G., 1999. A fast numerical scheme for computing the response of composites using grid refinement. Eur. Phys. J. AP 6, 41-47.

Gurson, A., 1977. Continuum theory of ductile rupture by void nucleation and growth: Part I - Yield criteria and flow rules for porous ductile media. J. Engng. Mat. Tech. 99, $1-15$.

Hodkin, E., 1980. The ratio of grain boundary energy to surface energy of nuclear ceramics as determined from pore geometries. J. Nucl. Mater. 88, 7 - 14 .

Idiart, M., Moulinec, H., Ponte Castañeda, P., Suquet, P., 2006. Macroscopic behavior and field fluctuations in viscoplastic composites: Second-order estimates versus full-field simulations. J. Mech. and Phys. Solids 54, 1029-1063.

Julien, J., Gărăjeu, M., Michel, J.-C., 2011. A semi-analytical model for the behavior of saturated viscoplastic materials containing two populations of voids of different sizes. Int. J. Solids Struct. 48 (10), 1485 - 1498.

Kanit, T., Forest, S., Galliet, I., Mounoury, V., Jeulin, D., 2003. Determination of the size of the representative volume element for random composites: statistical and numerical approach. Int. J. Solids Struc. 40, 3647-3679.

Kashibe, S., Une, K., 1991. Effects of temperature cycling and heating rate on fission gas release of bwr fuels. J. Nucl. Sci. Technol. 28 (1), 1090-1099.

Kashibe, S., Une, K., Nogita, K., 1993. Formation and growth of intragranular fission gas bubbles in $\mathrm{UO}_{2}$ fuels with burnup of 6-83 GWd/t. J. Nucl. Mater. 206 (1), $22-34$.

Killeen, J., 1980. Fission gas release and swelling in $\mathrm{UO}_{2}$ doped with $\mathrm{Cr}_{2} \mathrm{O}_{3}$. J. Nucl. Mat. $88(2-3), 177-184$.

Koplik, J., Needlemann, A., 1988. Void growth and coalescence in porous plastic solids. Int. J. Solids Struct. 24, 835-853.

Kröner, E., 1972. Statistical Continuum Mechanics. Springer-Verlag: Wien - New York.

Leblond, J., Perrin, G., Suquet, P., 1994. Exact results and approximate models for porous viscoplastic solids. Int. J. Plasticity 10, 213-235.

Lösönen, P., 2000. On the behavior of intragranular fission gas in $\mathrm{UO}_{2}$ fuel. J. Nucl. Mat. $280,56-72$.

Michel, J., Moulinec, H., Suquet, P., 1999. Effective properties of composite materials with periodic microstructure: a computational approach. Comp. Meth. Appl. Mech. Engng. 172, 109-143. 
Michel, J., Moulinec, H., Suquet, P., 2000. A computational method based on augmented Lagrangians and fast Fourier transforms for composites with high contrast. CMESComp. Model. Eng. 1, 79-88.

Michel, J., Moulinec, H., Suquet, P., 2001. A computational scheme for linear and nonlinear composites with arbitrary phase contrast. Int. J. Numer. Meth. Engng 52, 139-160.

Monchiet, V., Bonnet, G., 2012. A polarization-based FFT iterative scheme for computing the effective properties of elastic composites with arbitrary contrast. Int. J. Numer. Meth. Engng. 89, 1419-1436.

Moulinec, H., Silva, F., 2014. Comparison of three accelerated FFT-based schemes for computing the mechanical response of composite materials. Int. J. Numer. Meth. Engng. 97 (13), 960-985.

Moulinec, H., Suquet, P., 1994. A fast numerical method for computing the linear and nonlinear properties of composites. C. R. Acad. Sc. Paris II 318, 1417-1423.

Moulinec, H., Suquet, P., 1998. A numerical method for computing the overall response of nonlinear composites with complex microstructure. Comp. Meth. Appl. Mech. Engng. 157, 69-94.

Müller, D., 1956. A method for solving algebraic equations using an automatic computer. MTAC 10, 208-215.

Noirot, J., Noirot, L., Desgranges, L., Lamontagne, J., Blay, T., Pasquet, B., Muller, E., September 19-22 2004. Fission gas inventory in PWR high burnup fuel: Experimental characterization and modeling. In: Proceedings of the 2004 International Meeting on LWR Fuel Performance. Orlando, Florida, pp. 329 - 338.

Ponte Castañeda, P., 1991. The effective mechanical properties of nonlinear isotropic composites. J. Mech. and Phys. Solids 39, 45-71.

Ponte Castañeda, P., Zaidman, M., 1996. The finite deformation of nonlinear composite materials - I. instantaneous constitutive relations. Int. J. Solids Struc. 33 (9), 1271 1286.

Reynolds, G., Beeré, W., Sawbridge, P., 1971. The effect of fission products on the ratio of grain-boundary energy to surface energy in irradiated uranium dioxide. J. Nucl. Mater. $41(1), 112-114$.

Soulacroix, J., 2014. Approche micromécanique du comportement du combustible dioxyde d'uranium. Ph.D. thesis, Arts et Métiers ParisTech. 
Suquet, P., Moulinec, H., Castelnau, O., Montagnat, M., Lahellec, N., Grennerat, F., Duval, P., Brenner, R., 2012. Multi-scale modeling of the mechanical behavior of polycrystalline ice under transient creep. Procedia IUTAM 3, 76 - 90, IUTAM Symposium on Linking Scales in Computations: From Microstructure to Macro-scale Properties.

Tvergaard, V., 1990. Material failure by void growth to coalescence. Adv. Appl. Mech. 27, $83-151$.

Une, K., 1988. Simulated bubble swelling in $\mathrm{UO}_{2}$ pellet. J. Nucl. Mater. 158, $188-201$.

Vincent, P.-G., Monerie, Y., Suquet, P., 2008. Ductile damage of porous materials with two populations of voids. Comptes Rendus Mécanique 336, 245-259.

Vincent, P.-G., Monerie, Y., Suquet, P., 2009a. Porous materials with two populations of voids under internal pressure. I: Instantaneous constitutive relations. Int. J. Solids Struct. 46, 480-506.

Vincent, P.-G., Monerie, Y., Suquet, P., 2009b. Porous materials with two populations of voids under internal pressure: II. Growth and coalescence of voids. Int. J. Solids Struct. $46(3-4), 507-526$.

Vincent, P.-G., Suquet, P., Monerie, Y., Moulinec, H., 2014a. Effective flow surface of porous materials with two populations of voids under internal pressure: I. A GTN model. Int. J. Plasticity 56, $45-73$.

Vincent, P.-G., Suquet, P., Monerie, Y., Moulinec, H., 2014b. Effective flow surface of porous materials with two populations of voids under internal pressure: II. Full-field simulations. Int. J. Plasticity 56, 74-98.

White, R., 2004. The development of grain-face porosity in irradiated oxide fuel. J. Nucl. Mater. 325 (1), $61-77$.

Wilkins, M. L., 1964. Calculation of elastic-plastic flow. Vol. 3. (B. Alder et al., Eds.), Academic Press, New York.

Zacharie, I., Lansiart, S., Combette, P., Trotabas, M., Coster, M., Groos, M., 1998. Microstructural analysis and modelling of intergranular swelling of an irradiated $\mathrm{UO}_{2}$ fuel treated at high temperature. J. Nucl. Mater. 255 (2-3), $92-104$.

\section{Appendix A. Ellipsoidal and lenticular bubbles}

Figure A.21 shows a lenticular bubble and an ellipsoidal bubble. The revolution axis is the minor axis of the ellipse (or of the lens). These two bubbles are considered as equivalent if they have the same volume and the same surface on the grain boundary, i.e. if the semi-major-axis of the ellipse $b$ is equal to $r_{e}$. This implies that: 


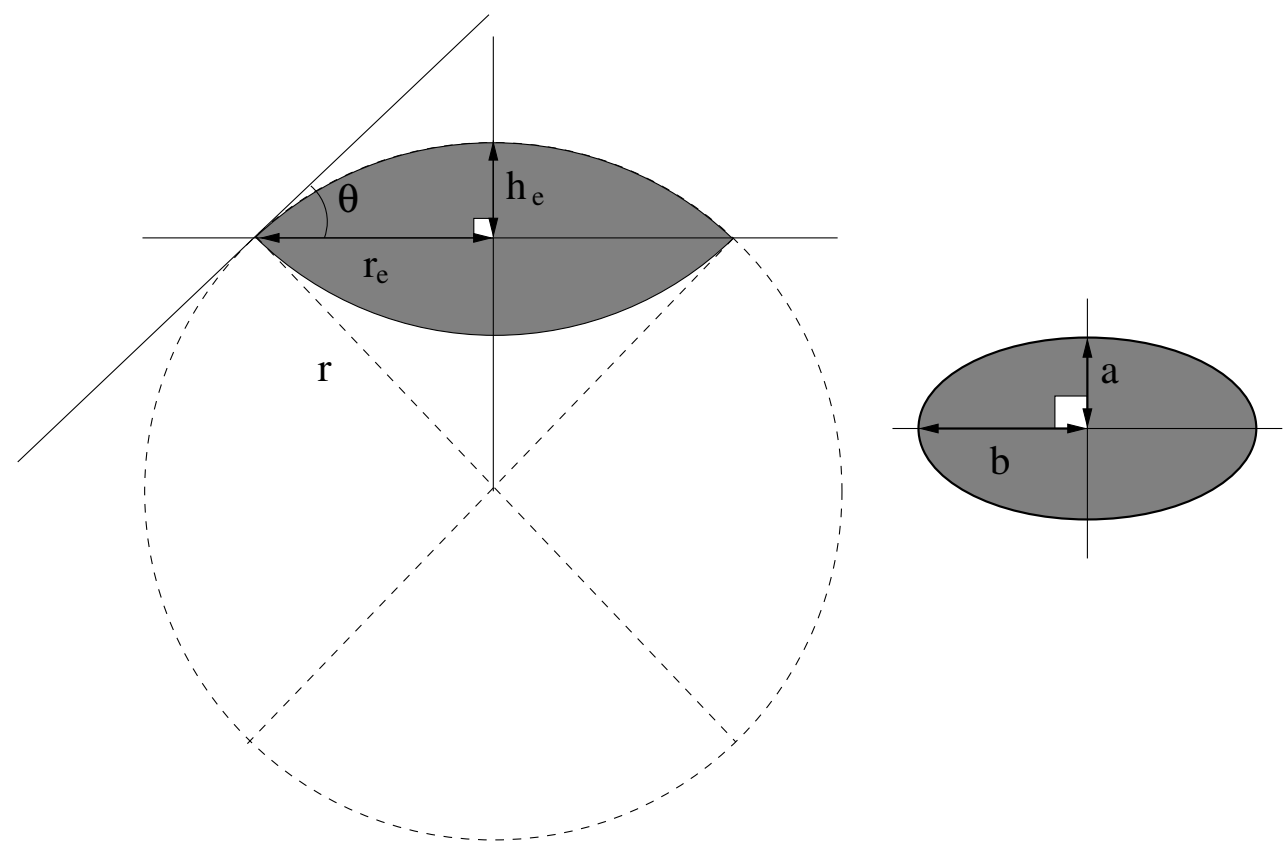

Figure A.21: Notations for a lenticular bubble and for an ellipsoidal bubble.

$$
\frac{2 \pi}{3} h_{e}^{2}\left(3 r-h_{e}\right)=\frac{4}{3} \pi b^{2} a .
$$

In that case, the aspect ratio of the ellipsoidal bubble (defined as $w_{e}=a / b$ ) reads as:

$$
w_{e}=\frac{1-\frac{3}{2} \cos (\theta)+\frac{1}{2} \cos ^{3}(\theta)}{\sin ^{3}(\theta)} .
$$

On the other hand, the aspect ratio of the lenticular bubble (defined as $w_{l}=h_{e} / r_{e}$ ) reads as:

$$
w_{l}=\frac{1-\cos (\theta)}{\sin (\theta)} .
$$

The specific value of the contact angle $\theta=45^{\circ}$ corresponds to $w_{e}=0.32$ and $w_{l}=0.41$.

\section{Appendix B. Deviation from isotropy}




\begin{tabular}{|c|c|c|c|c|c|c|}
\hline grains & $r_{e} / r_{g}$ & $f_{e}$ & $r_{s} / r_{e}$ & $\Sigma_{m} / \Sigma_{e q}=4$ & $\Sigma_{m} / \Sigma_{e q}=0$ & $\Sigma_{m} / \Sigma_{e q}=\infty$ \\
\hline \multirow{3}{*}{21} & \multirow{3}{*}{0.1} & \multirow{3}{*}{ sat } & 1.75 & 9.76 & 0.75 & 15.08 \\
\hline & & & 1.5 & 11.58 & 2.34 & 25.75 \\
\hline & & & 1.25 & 8.37 & 4.3 & 17.99 \\
\hline mean 21 & 0.1 & sat & 1.25 & 4.57 & 2.91 & 8.61 \\
\hline \multirow{3}{*}{21} & \multirow{3}{*}{0.16} & \multirow{3}{*}{ sat } & 1.75 & 12.18 & 1.25 & 19.62 \\
\hline & & & 1.5 & 15.32 & 2.08 & 21.9 \\
\hline & & & 1.25 & 6.89 & 4.34 & 17.55 \\
\hline \multirow{3}{*}{21} & \multirow{3}{*}{0.2} & \multirow{3}{*}{ sat } & 1.75 & 7.98 & 0.92 & 15.31 \\
\hline & & & 1.5 & 3.28 & 1.25 & 21.13 \\
\hline & & & 1.25 & 11.53 & 2.92 & 15.77 \\
\hline \multirow{3}{*}{80} & \multirow{3}{*}{0.16} & \multirow{3}{*}{ sat } & 1.75 & 2.23 & 0.48 & 10.27 \\
\hline & & & 1.5 & 5.46 & 0.72 & 9.72 \\
\hline & & & 1.25 & 8.83 & 2.02 & 11.21 \\
\hline \multirow{3}{*}{80} & \multirow{3}{*}{0.32} & \multirow{3}{*}{ sat } & 1.75 & 5.14 & 0.38 & 11.56 \\
\hline & & & 1.5 & 6.51 & 0.49 & 13.0 \\
\hline & & & 1.25 & 2.86 & 0.83 & 11.56 \\
\hline 80 & 0.32 & $1 \%$ & \multirow{5}{*}{1.75} & 5.26 & 0.31 & 29.47 \\
\hline 80 & 0.16 & $1 \%$ & & 3.27 & 0.1 & 9.37 \\
\hline 21 & 0.2 & $1 \%$ & & 10.66 & 0.68 & 31 \\
\hline 21 & 0.16 & $1 \%$ & & 12.18 & 1.25 & 19.62 \\
\hline 21 & 0.1 & $1 \%$ & & 9.33 & 0.43 & 22.65 \\
\hline 80 & 0.16 & $0.1 \%$ & \multirow{5}{*}{1.75} & 2.07 & 0.03 & 31.24 \\
\hline 80 & 0.32 & $0.1 \%$ & & 2.84 & 0.04 & 1.15 \\
\hline 21 & 0.2 & $0.1 \%$ & & 3.06 & 0.08 & 15.31 \\
\hline 21 & 0.16 & $0.1 \%$ & & 3.95 & 0.03 & 23.4 \\
\hline 21 & 0.1 & $0.1 \%$ & & 5.39 & 0.02 & 23.42 \\
\hline
\end{tabular}

Table B.5: Deviation from isotropy in \% measured for different configurations. "sat" indicates the case of saturated grains boundaries. 


\begin{tabular}{|c|c|c|c|c|}
\hline$\frac{\left|\Sigma_{m}\right|}{\Sigma_{e q}}$ & $\boldsymbol{\Sigma}=\left(\begin{array}{ccc}S & 0 & 0 \\
0 & S & 0 \\
0 & 0 & T\end{array}\right)|S| \leq|T|$ & \multicolumn{2}{|c|}{$\boldsymbol{\Sigma}=\left(\begin{array}{ccc}S & 0 & 0 \\
0 & S & 0 \\
0 & 0 & T\end{array}\right)|S| \geq|T|$} \\
\hline & load direction & $\begin{array}{c}\text { deviation } \\
\text { from isotropy }(\%)\end{array}$ & load direction & $\begin{array}{c}\text { deviation } \\
\text { from isotropy (\%) }\end{array}$ \\
\hline$\infty$ & $\mathrm{T}=\mathrm{S}$ & 21.13 & $\mathrm{~T}=\mathrm{S}$ & 21.13 \\
10 & $\mathrm{~T}=1.1 \mathrm{~S}$ & 7.34 & $\mathrm{~T}=0.91 \mathrm{~S}$ & 8.62 \\
4 & $\mathrm{~T}=1.27 \mathrm{~S}$ & 18.68 & $\mathrm{~T}=0.77 \mathrm{~S}$ & 3.28 \\
3.33 & $\mathrm{~T}=4 / 3 \mathrm{~S}$ & 2.69 & $\mathrm{~T}=0.727 \mathrm{~S}$ & 14.82 \\
2 & $\mathrm{~T}=1.6 \mathrm{~S}$ & 2.85 & $\mathrm{~T}=0.571 \mathrm{~S}$ & 1.68 \\
1.17 & $\mathrm{~T}=2.2 \mathrm{~S}$ & 1.91 & $\mathrm{~T}=1 / 3 \mathrm{~S}$ & 0.82 \\
1.0 & $\mathrm{~T}=2.5 \mathrm{~S}$ & 0.73 & $\mathrm{~T}=0.25 \mathrm{~S}$ & 1.05 \\
0.33 & $\mathrm{~S}=0, \mathrm{~T}=1$ & 0.68 & $\mathrm{~T}=-0.5 \mathrm{~S}$ & 1.07 \\
0 & $\mathrm{~T}=-2 \mathrm{~S}$ & 1.25 & & \\
\hline
\end{tabular}

Table B.6: Deviation from isotropy in \% measured for the microstructure (21 grains, saturated grain boundaries, $\left.r_{e} / r_{g}=0.2, r_{s} / r_{e}=1.5\right)$ for different loading directions. 